\title{
Identifying clinical clusters with distinct trajectories in first-episode psychosis through an unsupervised machine learning technique
}

\author{
Silvia Amoretti ${ }^{a, b, c, 1}$, Norma Verdolini ${ }^{b, c, 1}$, Gisela Mezquida ${ }^{a, b}$, \\ Francisco Diego Rabelo-da-Ponte ${ }^{d}$, Manuel J Cuesta ${ }^{e, f}$, \\ Laura Pina-Camacho ${ }^{\text {b,g }}$, Marta Gomez-Ramiro a, \\ Concepción De-la-Cámara ${ }^{a, h}$, Ana González-Pinto ${ }^{b, i}$, \\ Covadonga M. Díaz-Caneja ${ }^{\mathrm{b}, \mathrm{g}}$, Iluminada Corripio ${ }^{\mathrm{b}, \mathrm{j}}$, \\ Eduard Vieta ${ }^{\mathrm{b}, c, *}$, Elena de la Serna ${ }^{\mathrm{b}, \mathrm{k}}$, Anna Mané ${ }^{\mathrm{b}, \mathrm{l}, \mathrm{m}}$, \\ Brisa Soléb,c, André F Carvalho ${ }^{n}$, Maria Serra ${ }^{c}$, \\ Miguel Bernardo ${ }^{a, b}$
}

\footnotetext{
${ }^{a}$ Barcelona Clinic Schizophrenia Unit, Hospital Clinic of Barcelona, August Pi I Sunyer Biomedical Research Institute (IDIBAPS), Neuroscience Institute, University of Barcelona, Spain

${ }^{\mathrm{b}}$ Biomedical Research Networking Center for Mental Health Network (CIBERSAM), Spain

'Bipolar and Depressive Disorders Unit, Hospital Clinic, University of Barcelona, Institute of Neuroscience, IDIBAPS, CIBERSAM, 170 Villarroel st, 12-0, 08036, Barcelona, Catalonia, Spain

${ }^{\mathrm{d}}$ Laboratory of Molecular Psychiatry, Hospital de Clínicas de Porto Alegre, Federal University of Rio Grande do Sul, Brazil

e Department of Psychiatry, Complejo Hospitalario de Navarra, Pamplona, Spain

${ }^{\mathrm{f}}$ Navarra Institute for Health Research (IdiSNA), Pamplona, Spain

s Department of Child and Adolescent Psychiatry, Institute of Psychatry and Mental Health, Hospital General Universitario Gregorio Marañon, IiSGM, School of Medicine, Universidad Complutense, Madrid, Spain

${ }^{\mathrm{h}}$ Hospital Clínico Universitario and Instituto de Investigación Sanitaria (IIS), Department of Medicine and Psychiatry. Universidad de Zaragoza, Zaragoza, Spain

i Department of Psychiatry, Araba University Hospital, Bioaraba Research Institute, Department of Neurociences, University of the Basque Country, Vitoria, Spain

${ }^{j}$ Psychiatry Department, Institut d'Investigació Biomèdica-Sant Pau (IIB-SANT PAU), Hospital de la Santa Creu i Sant Pau; Universitat Autònoma de Barcelona (UAB), Barcelona, Spain
}

\footnotetext{
* Corresponding author at: Biomedical Research Networking Center for Mental Health Network (CIBERSAM), Spain E-mail address: EVIETA@clinic.cat (E. Vieta).

${ }^{1}$ The first two authors contributed equally to this work.
} 


\author{
${ }^{k}$ Department of Child and Adolescent Psychiatry and Psychology, Clínic Institute of Neurosciences, \\ Hospital Clínic de Barcelona, 2017SGR881, University of Barcelona, IDIBAPS, Barcelona, Spain \\ 'Hospital del Mar Medical Research Institute (IMIM), Barcelona, Spain \\ ${ }^{\mathrm{m}}$ Autonomous University of Barcelona (UAB), Barcelona, Spain \\ n Department of Psychiatry, University of Toronto, Toronto, ON, Canada; Centre for Addiction and \\ Mental Health (CAMH), Toronto, ON, Canada
}

Received 6 November 2020; received in revised form 4 January 2021; accepted 18 January 2021

Available online $x x x$

\section{KEYWORDS \\ First-episode psychosis; Symptomatology; Cognitive reserve; Functioning; Machine learning}

\begin{abstract}
The extreme variability in symptom presentation reveals that individuals diagnosed with a firstepisode psychosis (FEP) may encompass different sub-populations with potentially different illness courses and, hence, different treatment needs. Previous studies have shown that sociodemographic and family environment factors are associated with more unfavorable symptom trajectories. The aim of this study was to examine the dimensional structure of symptoms and to identify individuals' trajectories at early stage of illness and potential risk factors associated with poor outcomes at follow-up in non-affective FEP. One hundred and forty-four non-affective FEP patients were assessed at baseline and at 2-year follow-up. A Principal component analysis has been conducted to identify dimensions, then an unsupervised machine learning technique (fuzzy clustering) was performed to identify clinical subgroups of patients. Six symptom factors were extracted (positive, negative, depressive, anxiety, disorganization and somatic/cognitive). Three distinct clinical clusters were determined at baseline: mild; negative and moderate; and positive and severe symptoms, and five at follow-up: minimal; mild; moderate; negative and depressive; and severe symptoms. Receiving a low-dose antipsychotic, having a more severe depressive symptomatology and a positive family history for psychiatric disorders were risk factors for poor recovery, whilst having a high cognitive reserve and better premorbid adjustment may confer a better prognosis. The current study provided a better understanding of the heterogeneous profile of FEP. Early identification of patients who could likely present poor outcomes may be an initial step for the development of targeted interventions to improve illness trajectories and preserve psychosocial functioning.
\end{abstract}

(C) 2021 Elsevier B.V. and ECNP. All rights reserved.

\section{Introduction}

The extreme variability in the presentation of symptom suggests that people diagnosed with first-episode psychosis (FEP) may encompass distinct sub-populations of patients (Cocchi et al., 2014), with potentially different illness courses and, hence, different treatment needs (Colasanti et al., 2010). The identification of early clinical and sociodemographic features may be important in identifying subsets of patients with similar characteristics, facilitating personalised treatment approaches (Compton et al., 2014).

Two general models have been used so far to describe the varied clinical manifestations of psychosis, namely the categorical and dimensional approaches. The categorical model involves mutually exclusive classes, which are defined by specific patterns of symptoms. Thus, it considers illness as being either present or absent. In line with this, the current diagnostic systems, such as the Diagnostic and Statistical Manual of Mental Disorders (DSM-5) (American Psychiatric Association, 2013) and the International Statistical Classification of Diseases and Related Health Problems (ICD-10) (World Health Organization, 2004) adopt a categorical approach to classify psychotic disorders. On the other hand, the dimensional perspective proposes that symptoms of psychosis tend to cluster together within different symptom complexes or dimensions, which can co-exist in the same in- dividual and cut across different types of psychoses (Peralta and Cuesta, 2001). Thus, dimensions, unlike categories, are not mutually exclusive. Most dimensional studies observed 4 or 5 dimensions within psychosis, with positive, negative, disorganisation, affective and cognitive symptom domains most frequently reported (Potuzak et al., 2012). However, the number of dimensions identified has varied across studies depending on the nature of the assessment tools used and the heterogeneity of included participants (Malla and Payne, 2005; Rodriguez-Jimenez et al., 2013; Compton et al., 2014). This hampers the comparability of the studies and their translation into consistent, uniform and replicable data. Of note, a dimensional approach has been gradually incorporated in recent diagnostic classifications. For example, in the DSM-5 schizophrenia subtypes have been excluded and a severity rating of core symptoms has been included. Nonetheless, a mixed dimensional-categorical approach to psychosis, and particularly FEP psychopathology, is still lacking in the currently available versions of both DSM and ICD (Vieta, 2016; Stein et al., 2020). Furthermore, clustering algorithms as fuzzy algorithm may be clinically useful for identifying a spectrum in subjects with FEP, since it groups the subjects in a more natural way, not forcing a subject to belong to one or the other group, unlike other clustering methods (Sugranya and Shanthi, 2012; Wu et al., 2020). 
The symptoms trajectories among patients with FEP are also heterogeneous (Abdin et al., 2017). Previous studies have shown that sociodemographic factors including younger age, male gender, education and clinical factors such as having poor functioning at baseline or a longer duration of untreated psychosis (DUP) are associated with poor symptom trajectories (Austin et al., 2015; Verma et al., 2012). A meta-analysis revealed a significant association between DUP and several outcomes, including positive and negative symptoms, depression/anxiety, social functioning and global outcome (Perkins et al., 2005). Furthermore, poor premorbid adjustment and the early appearance of negative symptoms are also predictive of subsequent worse response to treatment (Malla and Payne, 2005; Rabinowitz et al., 2006). Numerous aspects related with the family environment, such as expressed emotions, have been shown to influence the progression of psychosis (Hinojosa-Marqués et al., 2019). Thus, these environmental factors have also been considered potential factors for the risk, onset and progression of psychosis (Varese et al., 2012). A strong relationship between cognitive performance and clinical improvement in FEP has also been described (Allott et al., 2011; Rodriguez-Sanchez et al., 2013). More specifically, in the field of cognition-related factors, higher cognitive reserve (CR) is associated with a later onset of psychosis and better recovery (Barnett et al., 2006). Thus, CR has been considered as a positive moderator of the impact of pathology on clinical course, functional outcome and cognitive performance (de la Serna et al., 2013; Amoretti et al., 2016, 2018, 2020).

Given their significant impact on both short and long-term outcomes such as psychosocial functioning and quality of life, identifying both baseline and follow-up clinical phenotypes, sociodemographic and family factors in FEPs could provide relevant information that might allow the definition of early personalised intervention strategies and tailored person-focused therapy. Consequently, the aims of this study were:

1. To examine the dimensional structure of psychopathological symptom presentation in FEP patients at different time points.

2. To identify clinical subgroups of patients based on the psychopathological dimensions at baseline and 2-year follow-up.

3. To search for differences between the different subgroups of patients in terms of sociodemographic, familial, clinical and psychosocial functioning.

4. To unravel the initial heterogeneity of psychopathological presentation by identifying subgroups with distinct longitudinal trajectories of disease.

\section{Experimental procedures}

\subsection{Sample}

The sample of this study came from a multicentre, naturalistic and longitudinal project "Phenotype-genotype interaction. Application of a predictive model in first psychotic episodes" (PEPs Project) (Bernardo et al., 2013, 2019), all under the umbrella of the Spanish Research Network on Mental Health (CIBERSAM) (Salagre et al., 2019). A total of 335 patients with a FEP were recruited from 16 centres located throughout the Spanish territory from April 2009 to April 2011. For the purpose of the present study, the final sample consisted of 144 FEP patients fulfilling the following inclusion criteria: 1 ) aged between 18 and 35 years at the time of first evaluation; 2) presence of psychotic symptoms of less than twelve months' duration; 3) ability to speak Spanish correctly and 4) signed informed consent. Exclusion criteria were: 1) mental retardation according to DSM-IV criteria; 2 ) history of head trauma with loss of consciousness and 3) physical disease with mental repercussions. To ensure more homogeneous sample diagnoses, for the current study we only included patients with non-affective FEP, as we considered affective FEP patients as a subgroup displaying several specific characteristics in terms of clinical course, functional outcome and antipsychotic treatments. We considered only FEP patients that have been diagnosed after 2 years of follow-up with a non-affective psychosis such as schizophrenia, schizophreniform, schizoaffective disorders and psychoses that are not otherwise specified, according to DSMIV-TR. In particular, only FEP patients with a complete psychopathological assessment at baseline and two-year follow-up have been included in the present study.

This study was conducted in accordance with the ethical principles of the Declaration of Helsinki and Good Clinical Practice and the Hospital Clinic Ethics and Research Board. All participants provided written informed consent prior to their inclusion in the study.

\subsection{Assessments}

\subsubsection{Clinical and sociodemographic assessment}

Clinical and sociodemographic data were systematically obtained for all participants and included: age, gender and parental socioeconomic status (SES). SES was determined using Hollingshead's Two-Factor Index of Social Position (Hollingshead and Redlich, 1958); pharmacological treatment was measured by chlorpromazine equivalents (CPZ) based on international consensus (Gardner et al., 2010); and the DUP was calculated as the number of days between the first manifestations of psychotic symptoms until the initiation of adequate treatment for psychosis. Drug misuse habits were also collected using a version of the European Addiction Severity Index (EuropASI) (KokkeviandHartgers, 1995).

Diagnoses at 2-year follow-up were determined with the Structured Clinical Interview for DSM (SCID-I-II) (First et al., 1997a, 1997b) according to DSM-IV criteria. Taking into consideration potential changes across time and in order to ensure diagnostic stability, the diagnoses of the patients who completed the study were determined based on information gathered at the two-year followup visit.

A psychopathological assessment was carried out with the PANSS (Kay et al., 1987), the Montgomery-Asberg Depression Rating Scale (MADRS) (Montgomery and Asberg, 1979) and the Clinical Global Impression Scale (CGI) (Guy, 1976). Higher scores indicate greater severity.

Traumatic Experiences in Psychiatric Outpatients Questionnaire (TQ) (Davidson and Smith, 1990)was used to assess for traumatic experiences. In this study we used the TQ dichotomous clinical cutoff scores that differentiate between the presence or absence of significant abuse and neglect.

\subsubsection{Family assessment}

The Family Environment was assessed by the Family Environment Scale (FES) (Moos and Moos, 1976). FES is a self-report instrument focused on the measurement and description of the interpersonal relationships among family members. FES evaluated family emotional climate in different categories: Cohesion (for mutual reliance); Expressivity (the extent to which family members express their feelings directly); Conflicts (for open expression of 
anger, aggressiveness and conflict); Independence (for an achievement orientated environment); Achievement Orientation (the extent to which family members are independent in their decisions); Intellectual-Cultural Orientation (for political, intellectual, cultural interests); Active-Recreational Orientation (for participation in social activities); Moral-Religious Emphasis (for the importance given to ethical and religious practices and values); Organisation (for the organization in activities and responsibilities); and Control (the extent to which the family considers rules and established procedures).

The family history of psychiatric disorders was assessed using a semi-structured interview. Then, patients were classified into two categories: 1) those with a first-degree relative with a psychiatric disorder, and 2) those patients who did not have a first-degree family member with a psychiatric disorder.

\subsubsection{Functional assessment}

The overall functional outcome was assessed by means of the Functioning Assessment Short Test (FAST) (Rosa et al., 2007). The FAST scale is used to evaluate 6 specific areas of functioning: autonomy, occupational functioning, cognitive functioning, financial issues, interpersonal relationships, and leisure time, and has been validated in FEP population (González-Ortega et al., 2010). Higher scores indicate worse functioning.

The Premorbid Adjustment Scale (PAS) (Cannon-Spoor et al., 1982) was applied retrospectively to assess premorbid adjustment. Higher scores indicate worse premorbid adjustment.

\subsubsection{Cognitive reserve assessment}

To assess CR we have used the three most commonly proposed proxy indicators of CR in psychiatry and particularly in FEP (Barnett et al., 2006; de la Serna et al., 2013; Amoretti et al., 2016, 2018, 2020) which include premorbid intelligence quotient (IQ), education and lifetime participation in leisure, social and physical activities. Because patients already experienced a FEP when we evaluated CR, we could only estimate premorbid variables. The estimated premorbid IQ was calculated with the vocabulary subtest of the Wechsler Adults Intelligence Scale (WAIS-III) as a measure reflecting crystallised intelligence. The vocabulary subtest seems to be stable during the progression of the disease and useful as a measure of estimated premorbid intelligence (de Oliveira et al., 2014). The second proxy, 'education' was assessed taking into account the number of years of obligatory education that subjects had completed as well as parents' educational level and the subject's lifetime school performance. Finally, lifetime participation in leisure, social and physical activities was assessed by PAS scale (scholastic performance) and by asking about involvement in social activities, ability to perform physical activities and enjoyment of hobbies. Higher scores correspond to better performance. A Principal Components Analysis (PCA) was performed to create a "Cognitive reserve score" for each subject with the three main proxies.

\subsection{Analysis}

\subsubsection{Pre-processing}

A PCA was carried out with the aim of assembling items of PANSS and MADRS in a series of coherent components that would be able to tackle psychopathological dimensions in patients with FEP at baseline and 2-year follow-up. Items with an unclear factor loading distribution (i.e., showing similar factor loadings in more than one component) or with low factor loadings $(<0.45)$ were excluded from the components. Considering the high correlation among items, we used an oblique rotation (Loo, 1979). The oblique rotation method allows the extent to which the factors are actually correlated to each other to be examined, and therefore renders it more realistic theoretically and empirically (Peralta and Cuesta, 2001).

\subsubsection{Unsupervised machine learning technique}

The machine learning analysis was performed using R software (version R 3.6.3) and RStudio (version 3.5.3). We performed the fuzzy analysis clustering to identify clinical subgroups of subjects with FEP at baseline and 2-year follow-up based on the psychopathological dimensions detected in the PCA (Lefort-Nesmard et al., 2018), using the R package "cluster" (version 2.1.0). The optimal number of clusters was determined by the Bayesian Information Criterion (BIC) (Fraley and Raftery, 1998). We performed Dann and Gamma Indexes to verify the quality of clustering at baseline as well as at follow-up. Furthermore, we performed a Discriminant function analysis (DFA) using package 'MASS' (version 7.3-53) at baseline and at 2-year follow-up to confirm the clusters retained and to investigate the predictive power of the clustering of each subject's psychopathological dimensions to the clinical cluster (Jensen et al., 2015).

Overall, there is two clustering methods: hard and soft (fuzzy) clustering. The former divided subjects into distinct subgroups, where each subject belongs exclusively to a single group, while the latter the subject may belong to more than one group, which may indicate a continuity or developmental aspect of a disease. In the fuzzy clustering, subjects at the boundaries between multiple clusters are not forced to belong entirely to one of the classes, but are instead assigned degrees of association between 0 and 1 , indicating their partial association. This type of grouping is much more natural than other methods since there are situations in which a subject may have mixed characteristics (Suganya and Shanthi, 2012).

Furthermore, we performed a discriminant function analysis using package 'MASS' (version 7.3-53) to confirm the clusters retained and to investigate the predictive power of the clustering of each participant's cognitive domain to the cognitive subgroup (Jensen et al., 2015).

\subsubsection{Statistical analysis}

Descriptive analyses identifying differences between the clusters and trajectories were conducted using chi-square for categorical variables. Group differences were examined using unpaired t-tests for normally distributed variables, or using Mann-Whitney $U$ tests for non-normal data. When comparing groups based on clinical, sociodemographic and functional variables, analysis of variance (ANOVA) was used to show the overall differences between groups with post-hoc Turkey's HSD test for multiple comparisons. To compare the performance at baseline and two-year follow-up, a repeated measures analysis of variance (ANOVA) was used.

Finally, we decided to identify trajectories of disease. Fourtrajectory groups were described as "excellent prognosis", "remitting course", "clinical worsening" and "chronic course". Patients with an excellent prognosis trajectory were those who exhibited mild symptoms at baseline and minimal or mild symptoms at followup, whereas those with remitting course had moderate or severe symptoms at baseline and minimal or mild symptoms at follow-up. Patients with a clinical worsening trajectory exhibited mild symptoms at baseline and negative/depressive or severe symptoms at follow-up. Finally, patients with a chronic course trajectory showed moderate/severe symptoms at baseline and negative/depressive or severe symptoms at follow-up.

Data were analysed using SPSS, version 23 . We considered $p<0.05$ as statistically significant.

\section{Results}

\subsection{Sociodemographic characteristics of the sample}

A total of 144 patients with first-episode non-affective psychosis were included in the present study and were re-evaluated at 2-year follow-up. Sixty-seven percent were 
male with a mean age of $25.26 \pm 5.13$ years (age at onset of psychosis was $24.91 \pm 5.14$ years). The mean dose of antipsychotic medication (prescribed by the psychiatrist) at baseline was equivalent to $619.72 \pm 422.26 \mathrm{mg} /$ day of $\mathrm{CPZ}$ (dose range 0 to $2400 \mathrm{mg} /$ day) and $201.71 \pm 279.98 \mathrm{mg} /$ day at follow-up (range, 0 to $1350 \mathrm{mg} / \mathrm{day}$ ). The mean DUP was determined as $102.26 \pm 126.81$ days (approximately 15 weeks). After 2 years of follow-up, the severity of clinical symptoms improved (values of partial eta squared range from 0.217 to 0.422 , medium to large effect sizes), as did functional scores $(\eta 2=0.169)$ (see Supplementary Table 1).

\subsection{Dimensional structure of symptoms}

Concerning the PCA at baseline the Kaiser-Meyer-Olkin (KMO) value, a measure of how suited the data is for factor analysis, was 0.844 and 0.876 at follow-up, exceeding the recommended value of 0.6 , and Bartlett's Test of Sphericity reached statistical significance, supporting the factorability of the correlation matrix. The six factors extracted at different time points are presented in Supplementary Table 2 and 3 accordingly. Factors were defined as "Disorganisation", "Negative”, “Depressive”, "Anxiety”, "Somatic/Cognitive" and "Positive". When we compared the PCA at baseline and at two-year follow-up, the PCA displayed the same components, showing the excellent stability of these six components. However, the components do not have exactly the same composition at both times of the evaluations and the PCA item loadings differed between baseline and follow-up.

\subsection{Clinical subgroups of patients at baseline and follow-up}

In relation to clustering performance, the PAM algorithm achieved an average Silhouette width equal to 0.71 , and Dunn index equal to 0.75 at baseline. At 2-year follow-up, the measures were 0.77 , and 0.80 , respectively. The Silhouette indicates how well objects are clustered, ranging from -1 (poorly) to 1 (well clustered). The Dunn index provides the ratio between compactness within a cluster and separation between different clusters (Hassani and Seidl, 2017; Tomasini et al., 2017).

The fuzzy algorithm through BIC method determined three distinct patient clusters at baseline: "mild symptoms" (39\%), "negative and moderate symptoms" (31\%) and "positive and severe symptoms" (30\%) (see Fig. 1a). In relation to clustering performance, it achieved a Dunn index equal to 0.79 and Gamma Index of 0.81 . For these indexes, the closer to 1 the better the grouping (Hassani and Seidl, 2017; Tomasini et al., 2017). At follow-up, five distinct clusters were found: "minimal" (34\%), "mild" (26\%), "moderate" (13\%), "negative and depressive" (13\%) and "severe symptoms" (14\%) (Fig. 1b). The clustering performance achieved a Dunn index of 0.75 and Gamma Index of 0.78 .

Additionally, the discriminant function analysis (DFA) exhibited two discriminant functions, which explained $91.8 \%$ and $8.2 \%$ of the variance, respectively (Wilks' $\lambda=0.14$, $\chi 2(12)=271.58, \quad p<0.001 ;$ Wilks' $\lambda=0.72, \quad \chi 2(5)=43.96$, $p<0.001)$ at the baseline. The subjects were correctly classified by DFA into $93.1 \%$ of the cases, demonstrating the validity of the three clinical clusters. While at 2years follow-up, the DFA showed the presence of three significant discriminant functions, which explained $89.9 \%$, $6.1 \%, 3.8 \%$ of the variance, respectively (Wilks' $\lambda=0.036$, $\chi 2(24)=455.39, p<0.001$; Wilks' $\lambda=0.407, \chi 2(15)=123.75$, $p<0.001$, Wilks' $\lambda=0.689, \chi 2(8)=51.24, p<0.001)$. The subjects were correctly classified by DFA into $94 \%$ of the cases, that is, it confirms the presence of the five clinical clusters.

When comparing the clinical and sociodemographic profiles, patients with mild symptoms had lower DUP, lower dose of antipsychotic, higher premorbid adjustment (PAS), lower illness severity, lower symptoms and greater psychosocial functioning (FAST) at baseline. There were no differences between clusters in terms of age, gender, SES, age of onset, alcohol or cannabis use, family and history of traumatic events/experiences (see Table 1). Patients with positive and severe symptoms reported to have a higher consume of alcohol (monthly alcohol consumption).

At 2-year follow-up patients with minimal symptoms had better premorbid adjustment, greater family cohesion and lower conflicts, more intellectual-cultural and activerecreational orientation, lower illness severity, lower symptoms and greater functioning. Sociodemographic and clinical characteristics and psychosocial functioning among clusters at 2-year follow-up are presented in Table 2.

\subsection{Trajectories of disease}

Four trajectory groups were determined as "excellent prognosis" (32\%), "remitting course" (37\%), "clinical worsening" (9\%) and "chronic course" (22\%) (see Fig. 2). A moderate symptoms group was excluded because it did not clearly differ from either the minimal and mild symptoms group or the negative and moderate, and severe symptoms group. The demographic and clinical characteristics of each trajectory and statistical comparisons between them are presented in Table 3.

Twenty percent of those patients who exhibited mild symptoms at baseline presented "negative and depressive" or "severe" symptoms at follow-up (clinical worsening group). This group differed from those who remained with "minimal" or "mild" symptoms (excellent prognosis) on lower rates of CPZ $(t=2.01 ; p=0.050)$, higher depressive symptoms $(t=-2.05 ; 0.046)$ and positive family history of psychiatric disorders $\left(\chi^{2}=7.60, p=0.022\right)$.

Sixty-two percent of patients with "moderate" or "severe" symptoms at baseline presented a remitting course. They showed better premorbid adjustment $(t=-2.91$; $p=0.005)$ and higher CR $(t=2.13 ; 0.037)$ compared to those with a chronic course.

Finally, those patients who showed "minimal" or "mild" symptoms (excellent prognosis + remitting course) had better premorbid adjustment, higher family expression and active-recreational orientation at baseline compared to those who presented "negative and depressive" or "severe" symptoms at follow-up (clinical worsening + chronic course). At two-year follow-up, they also showed greater family cohesion, expression and active-recreational orientation, lower illness severity, lower symptoms and greater functioning (sociodemographic and clinical characteristics of these groups are presented in Supplementary Table 4). 
A
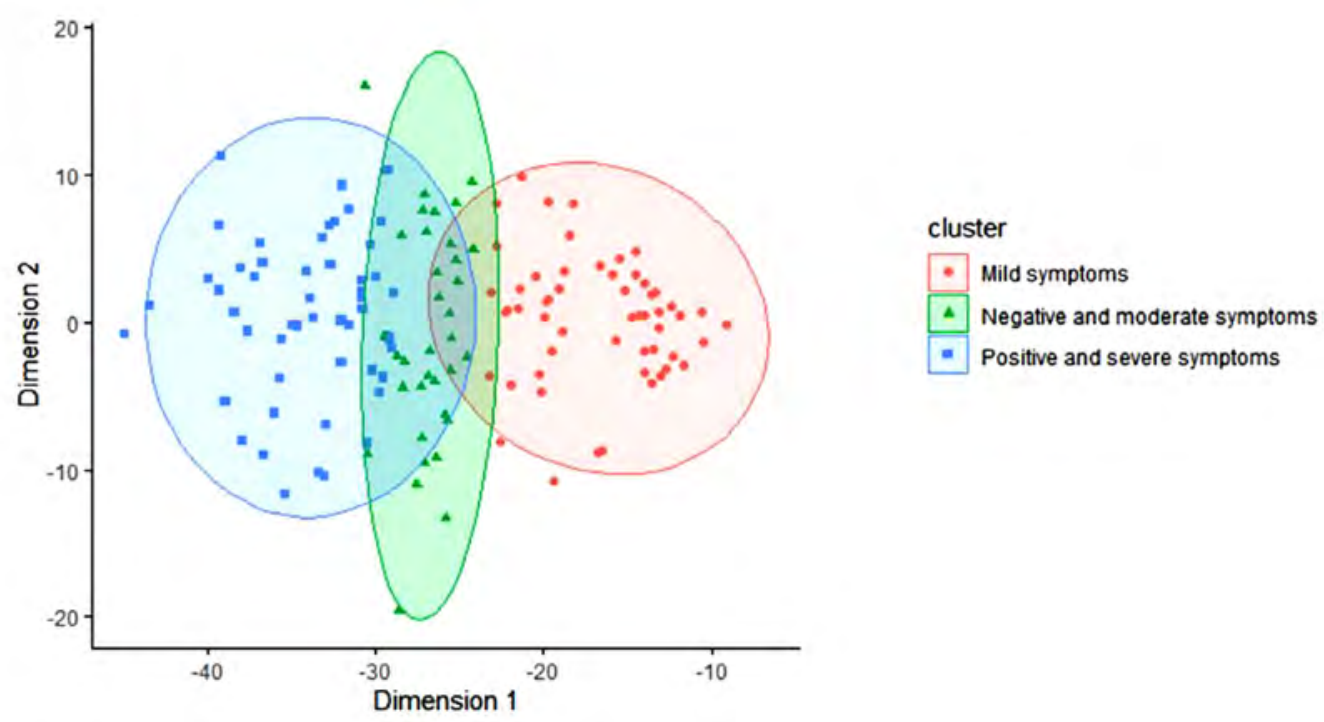

B

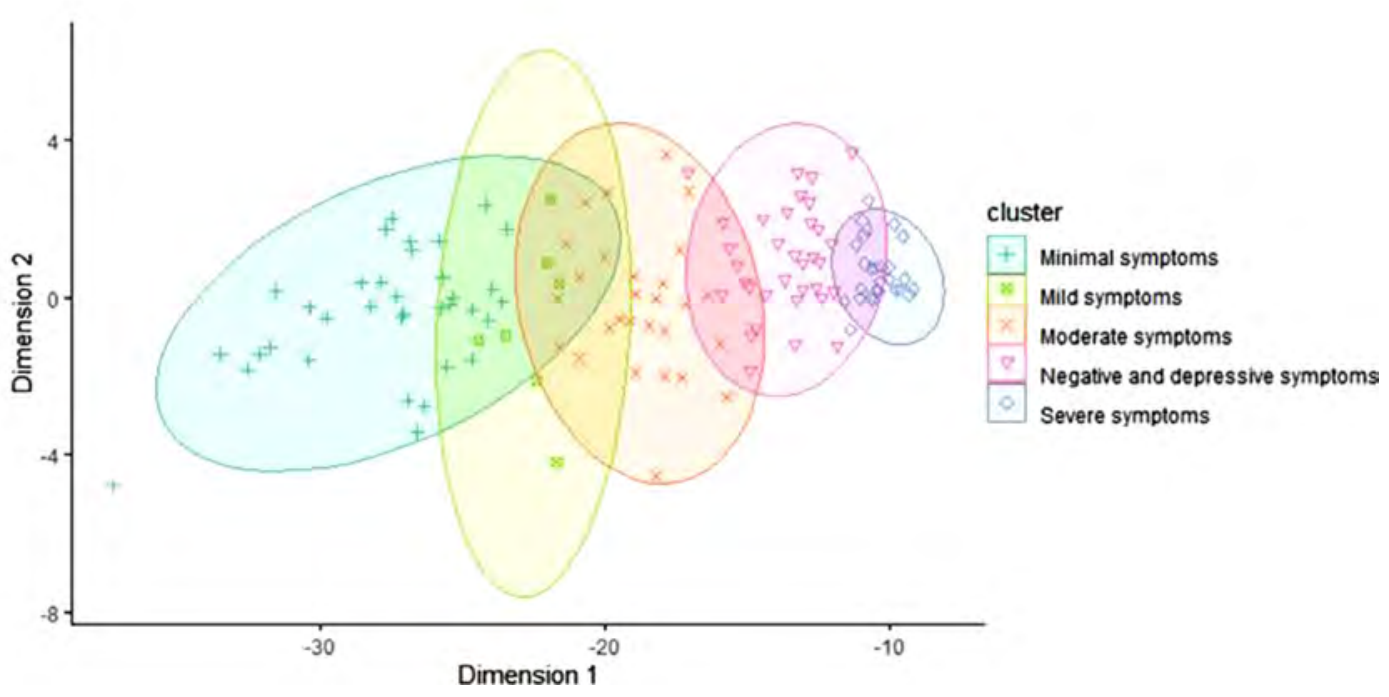

Fig. 1 Distribution of clinical subgroups of subjects with first-episode psychosis at baseline and at 2-years using fuzzy clustering algorithm

A) Cluster distribution at the baseline. B) Cluster distribution at the 2-year follow-up. Each axis means a reduction of symptomatology domains and each point is a subject.

\begin{tabular}{|c|c|c|c|c|c|c|c|c|c|c|}
\hline \multirow[b]{2}{*}{ Excellent prognosis } & \multirow[b]{2}{*}{$(32 \%)$} & & & & \multicolumn{5}{|c|}{ Follow-up } & \multirow[b]{2}{*}{ Total } \\
\hline & & & & & $\begin{array}{l}\text { Minimal } \\
\text { symptoms }\end{array}$ & $\begin{array}{c}\text { Mild } \\
\text { symptoms }\end{array}$ & $\begin{array}{l}\text { Moderate } \\
\text { symptoms }\end{array}$ & $\begin{array}{l}\text { Negative and } \\
\text { depressive } \\
\text { symptoms }\end{array}$ & $\begin{array}{c}\text { Severe } \\
\text { symptoms }\end{array}$ & \\
\hline Remitting course & $(37 \%)$ & \multirow{3}{*}{ 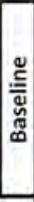 } & Mild symptoms & \multirow{3}{*}{$\begin{array}{c}\text { Count (\% } \\
\text { within } \\
\text { baseline) }\end{array}$} & $\begin{array}{c}26 \\
(46.40 \%)\end{array}$ & $\begin{array}{c}14 \\
(25.00 \%)\end{array}$ & $\begin{array}{c}5 \\
(8.90 \%)\end{array}$ & $\begin{array}{c}3 \\
(5.40 \%)\end{array}$ & $\begin{array}{c}8 \\
(14.30 \%)\end{array}$ & 56 \\
\hline Clinical worsening & $(9 \%)$ & & $\begin{array}{c}\text { Negative and } \\
\text { moderate symptoms }\end{array}$ & & $\begin{array}{c}11 \\
(24.40 \%)\end{array}$ & $\begin{array}{c}10 \\
(22.20 \%)\end{array}$ & $\begin{array}{c}10 \\
(22.20 \%)\end{array}$ & $\frac{9}{20.00 \times 1}$ & $\frac{5}{121.10 \%)}$ & 45 \\
\hline \multirow[t]{2}{*}{ Chranic caurse } & $(22 \%)$ & & $\begin{array}{c}\text { Positive and severe } \\
\text { symptoms }\end{array}$ & & $\begin{array}{c}12 \\
(27.90 \%)\end{array}$ & $\begin{array}{c}13 \\
(30.20 \%)\end{array}$ & $\begin{array}{c}4 \\
(9.30 \%)\end{array}$ & $\begin{array}{l}7 \\
\text { (16.30s) }\end{array}$ & $\begin{array}{c}7 \\
(16.30 \%)\end{array}$ & 43 \\
\hline & & & \multicolumn{2}{|l|}{ Total Count } & 49 & 37 & 19 & 19 & 20 & 144 \\
\hline
\end{tabular}

Fig. 2 Trajectories of symptoms in first-episode psychosis. 
Table 1 Baseline sociodemographic, clinical, functional and cognitive performance among clusters.

\begin{tabular}{|c|c|c|c|c|c|c|c|}
\hline & & $\begin{array}{l}\text { Mild symptoms } \\
(n=56)[1]\end{array}$ & $\begin{array}{l}\text { Negative and } \\
\text { moderate symptoms } \\
(n=45)[2]\end{array}$ & $\begin{array}{l}\text { Positive and } \\
\text { severe symptoms } \\
(n=43)[3]\end{array}$ & $\mathrm{F}$ & $P$ & $\begin{array}{l}\text { Post hoc } \\
\text { (Tukey's HSD) }\end{array}$ \\
\hline \multicolumn{8}{|l|}{ Sociodemographic variables } \\
\hline Gender: Male N(\%) & $37(66)$ & $32(71)$ & $27(63)$ & $\chi^{2}=0.70$ & 0.705 & - & \\
\hline Age $(M \pm S D)$ & $25.00 \pm 5.14$ & $25.16 \pm 5.33$ & $25.70 \pm 4.98$ & 0.24 & 0.790 & - & \\
\hline \multirow[t]{5}{*}{ SES (\%) } & High & $14(25)$ & $10(22)$ & $8(19)$ & $\chi^{2}=6.01$ & 0.815 & - \\
\hline & Medium-High & $5(9)$ & $5(11)$ & $4(9)$ & & & \\
\hline & Medium & $15(27)$ & $7(16)$ & $12(28)$ & & & \\
\hline & Medium-Low & $15(27)$ & $17(38)$ & $16(37)$ & & & \\
\hline & Low & $6(10)$ & $6(13)$ & $3(7)$ & & & \\
\hline DUP & $74.79 \pm 81.61$ & $143.89 \pm 180.57$ & $89.76 \pm 84.97$ & 3.86 & 0.024 & {$[1]<[2]^{*}$} & \\
\hline Age of onset & $24.75 \pm 5.14$ & $24.60 \pm 5.19$ & $25.46 \pm 4.79$ & 0.34 & 0.709 & - & \\
\hline $\mathrm{CPZ}$ & $460.92 \pm 354.99$ & $714.33 \pm 456.64$ & $723.04 \pm 412.74$ & 6.59 & 0.002 & {$[1]<[2-3]^{*}$} & \\
\hline Alcohol: Yes N (\%) & $30(46)$ & $25(56)$ & $30(70)$ & $\chi^{2}=2.96$ & 0.227 & - & \\
\hline Monthly alcohol & $22.70 \pm 38.60$ & $22.47 \pm 49.33$ & $64.77 \pm 96.85$ & 6.488 & 0.002 & {$[1-2]<[3]^{*}$} & \\
\hline Cannabis: Yes $N(\%)$ & $22(39)$ & $23(51)$ & $23(54)$ & $\chi^{2}=2.37$ & 0.306 & - & \\
\hline Monthly cannabis & $35.88 \pm 62.73$ & $62.96 \pm 125.56$ & $27.05 \pm 41.05$ & 2.262 & 0.108 & - & \\
\hline Trauma: Yes N (\%) & $34(61)$ & $24(53)$ & $27(49)$ & $\chi^{2}=2.58$ & 0.630 & - & \\
\hline PAS & $38.96 \pm 20.01$ & $52.28 \pm 24.42$ & $45.86 \pm 19.33$ & 4.52 & 0.013 & {$[1]<[2]^{*}$} & \\
\hline Cognitive Reserve & $74.62 \pm 12.63$ & $72.94 \pm 10.07$ & $77.32 \pm 11.29$ & 1.46 & 0.235 & - & \\
\hline \multicolumn{8}{|l|}{ Family } \\
\hline History: Yes N (\%) & $13(23)$ & $11(24)$ & $9(21)$ & $\chi^{2}=0.16$ & 0.924 & & \\
\hline Cohesion (C) & $49.74 \pm 7.51$ & $47.76 \pm 11.42$ & $48.54 \pm 7.89$ & $48.77 \pm 8.97$ & 0.562 & - & \\
\hline Expressivity (EX) & $50.83 \pm 9.66$ & $49.44 \pm 10.10$ & $47.67 \pm 10.06$ & $49.47 \pm 9.93$ & 0.322 & - & \\
\hline Conflicts (CON) & $48.62 \pm 7.41$ & $51.29 \pm 9.90$ & $51.13 \pm 7.86$ & $50.18 \pm 8.41$ & 0.221 & - & \\
\hline Independence (IND) & $49.57 \pm 9.16$ & $51.02 \pm 9.38$ & $48.18 \pm 9.15$ & $49.61 \pm 9.22$ & 0.389 & - & \\
\hline Achievement orientation $(\mathrm{AO})$ & $45.96 \pm 10.30$ & $47.17 \pm 8.38$ & $49.33 \pm 8.67$ & $47.32 \pm 9.31$ & 0.229 & - & \\
\hline $\begin{array}{l}\text { Intellectual-cultural } \\
\text { orientation (ICO) }\end{array}$ & $46.92 \pm 9.67$ & $49.05 \pm 10.30$ & $46.51 \pm 11.14$ & $47.46 \pm 10.29$ & 0.487 & - & \\
\hline
\end{tabular}


Table 1 (continued)

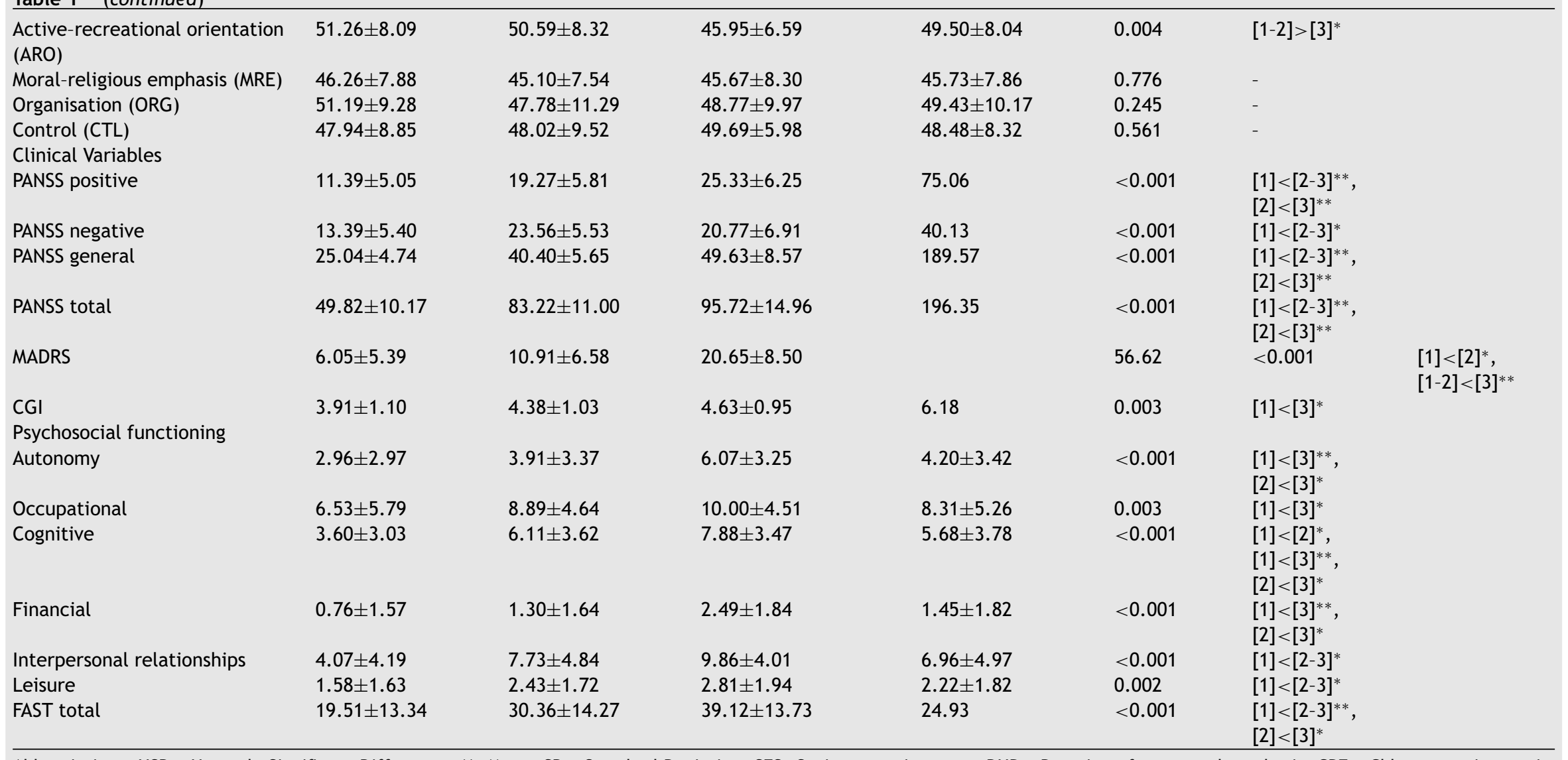

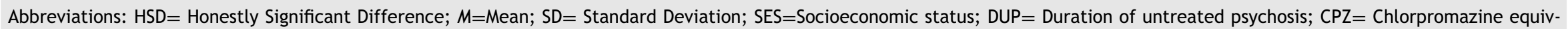
alents; PAS= Premorbid Adjustment Scale; PANSS= Positive and Negative Symptom Scale; MADRS= Montgomery-Asberg Depression Rating Scale; CGI=Clinical Global Impression Scale; FAST=Functioning Assessment Short Test. Significant differences $(p<0.05)$ marked in bold. ${ }^{*} p<0.05,{ }^{* *} p<0.001$. 
Table 2 Sociodemographic and clinical characteristics and psychosocial functioning among clusters at 2-year follow-up.

\begin{tabular}{|c|c|c|c|c|c|c|c|c|}
\hline & $\begin{array}{l}\text { Minimal } \\
\text { symptoms } \\
(n=49)[1]\end{array}$ & $\begin{array}{l}\text { Mild symptoms } \\
(n=37)[2]\end{array}$ & $\begin{array}{l}\text { Moderate } \\
\text { symptoms } \\
(n=19)[3]\end{array}$ & $\begin{array}{l}\text { Negative and } \\
\text { depressive } \\
\text { symptoms } \\
(n=19)[4]\end{array}$ & $\begin{array}{l}\text { Severe } \\
\text { symptoms } \\
(n=20)[5]\end{array}$ & $\mathrm{F}$ & $p$ & $\begin{array}{l}\text { Post hoc (Tukey's } \\
\text { HSD) }\end{array}$ \\
\hline \multicolumn{9}{|l|}{ Sociodemographic variables } \\
\hline Gender: Male N(\%) & $34(69)$ & $25(68)$ & $12(63)$ & $13(68)$ & $12(60)$ & $\chi^{2}=0.71$ & 0.950 & - \\
\hline Age $(M \pm S D)$ & $25.18 \pm 5.21$ & $25.78 \pm 4.75$ & $22.89 \pm 4.86$ & $24.68 \pm 5.55$ & $27.25 \pm 4.91$ & 1.98 & 0.101 & - \\
\hline \multirow{6}{*}{ SES (\%) } & High & $13(27)$ & $8(22)$ & $4(21)$ & $2(11)$ & $5(25)$ & $\chi^{2}=17.76$ & $0.603-$ \\
\hline & Medium-High & $4(8)$ & $4(11)$ & $3(16)$ & $2(11)$ & $1(5)$ & & \\
\hline & Medium & $14(29)$ & $9(24)$ & $5(26)$ & $1(5)$ & $5(25)$ & & \\
\hline & Medium-Low & $11(22)$ & $13(35)$ & $5(26)$ & $10(53)$ & $9(45)$ & & \\
\hline & Low & $6(12)$ & $3(8)$ & $2(11)$ & $4(21)$ & $0(0)$ & & \\
\hline & Missing & $1(2)$ & $0(0)$ & $0(0)$ & $0(0)$ & $0(0)$ & & \\
\hline DUP & $78.33 \pm 90.22$ & $98.29 \pm 94.64$ & $183.50 \pm 238.38$ & $87.50 \pm 110.37$ & $111.84 \pm 120.82$ & 2.22 & 0.071 & $\begin{array}{l}{[1]<[3]^{*} \text { ANOVA }} \\
\text { not significant, } \\
\text { Post-hoc test } \\
\text { significant }\end{array}$ \\
\hline Age of onset & $24.75 \pm 5.34$ & $25.58 \pm 4.66$ & $22.33 \pm 5.05$ & $24.42 \pm 5.33$ & $26.85 \pm 4.80$ & 2.12 & 0.082 & - \\
\hline $\mathrm{CPZ}$ & $150.21 \pm 222.16$ & $188.67 \pm 240.03$ & $297.63 \pm 414.53$ & $193.16 \pm 295.99$ & $269.04 \pm 298.57$ & 1.30 & 0.275 & - \\
\hline Alcohol: Yes N (\%) & $29(59)$ & $24(65)$ & $10(53)$ & $13(68)$ & $9(45)$ & $\chi^{2}=3.164$ & 0.531 & - \\
\hline Monthly alcohol & $25.80 \pm 51.47$ & $51.35 \pm 85.49$ & $42.05 \pm 74.52$ & $16 \pm 25.23$ & $40 \pm 76.96$ & $\chi^{2}=1.264$ & 0.287 & - \\
\hline Alcohol FUP & $29(59)$ & $24(65)$ & $10(53)$ & $12(63)$ & $8(40)$ & $\chi^{2}=5.90$ & 0.658 & - \\
\hline Monthly alcohol FUP & $25.73 \pm 41.73$ & $22.24 \pm 36.02$ & $34.42 \pm 89.84$ & $32.16 \pm 50.34$ & $12.40 \pm 30.31$ & $\chi^{2}=0.630$ & 0.642 & - \\
\hline Cannabis: Yes N (\%) & $22(45)$ & $18(49)$ & $9(47)$ & $12(63)$ & 7 (35) & $\chi^{2}=3.271$ & 0.513 & - \\
\hline Monthly cannabis & $27.33 \pm 48.82$ & $39.97 \pm 63.44$ & $37.11 \pm 56.89$ & $41.11 \pm 55.86$ & $85.05 \pm 176.27$ & $\chi^{2}=1.738$ & 0.145 & - \\
\hline Cannabis FUP & $8(32)$ & $5(14)$ & $5(26)$ & $4(21)$ & $3(15)$ & $\chi^{2}=3.67$ & 0.886 & - \\
\hline Monthly cannabis FUP & $8.76 \pm 26.52$ & $11 \pm 34.23$ & $2.74 \pm 7.16$ & $23.68 \pm 49.57$ & $11.95 \pm 32.67$ & $\chi^{2}=1.136$ & 0.342 & - \\
\hline Trauma: Yes N (\%) & $25(32)$ & $22(60)$ & $14(74)$ & $9(47)$ & $9(45)$ & $\chi^{2}=8.69$ & 0.371 & - \\
\hline PAS & $40.53 \pm 19.69$ & $39.64 \pm 18.34$ & $53.89 \pm 25.49$ & $54.63 \pm 22.13$ & $48.84 \pm 24.20$ & 2.97 & 0.022 & $\begin{array}{l}\text { Post hoc not } \\
\text { significant }\end{array}$ \\
\hline Cognitive reserve & $\begin{array}{l}76.83 \pm 12.41 \\
\text { Family }\end{array}$ & $76.22 \pm 9.68$ & $72.18 \pm 10.99$ & $72.59 \pm 12.26$ & $72.25 \pm 12.37$ & 1.10 & 0.358 & - \\
\hline History: Yes N (\%) & $11(22)$ & $6(16)$ & $3(16)$ & $4(21)$ & $9(45)$ & $\chi^{2}=7.05$ & 0.133 & - \\
\hline Cohesion (C) & $54.58 \pm 7.79$ & $51.36 \pm 9.12$ & $43.38 \pm 10.37$ & $47.43 \pm 8.86$ & $50.75 \pm 13.04$ & 4.37 & 0.003 & {$[1]>[3]^{*}$} \\
\hline Expressivity (EX) & $52.90 \pm 7.79$ & $54.68 \pm 8.56$ & $48.15 \pm 12.62$ & $47.71 \pm 9.43$ & $47.38 \pm 9.96$ & 2.45 & 0.051 & - \\
\hline Conflicts (CON) & $47.38 \pm 5.17$ & $47.32 \pm 4.62$ & $54.69 \pm 10.21$ & $48.29 \pm 7.41$ & $46.00 \pm 4.04$ & 4.13 & 0.004 & $\begin{array}{l}{[1-2]<[3]^{*}} \\
{[3]<[5]^{*}}\end{array}$ \\
\hline Independence (IND) & $53.25 \pm 8.82$ & $54.44 \pm 7.90$ & $51.23 \pm 9.88$ & $49.93 \pm 10.59$ & $51.88 \pm 7.95$ & 0.71 & 0.584 & - \\
\hline Achievement orientation (AO) & $48.58 \pm 9.28$ & $44.80 \pm 8.20$ & $43.69 \pm 8.62$ & $44.93 \pm 9.07$ & $43.38 \pm 13.80$ & 1.27 & 0.288 & - \\
\hline $\begin{array}{l}\text { Intellectual-cultural orientation } \\
\text { (ICO) }\end{array}$ & $53.48 \pm 11.29$ & $49.52 \pm 11.94$ & $41.92 \pm 10.36$ & $44.57 \pm 9.07$ & $51.00 \pm 13.96$ & 3.41 & 0.012 & {$[1]<[3]^{*}$} \\
\hline \multirow[t]{2}{*}{$\begin{array}{l}\text { Active-recreational orientation } \\
\text { (ARO) }\end{array}$} & $55.23 \pm 8.36$ & $53.36 \pm 11.28$ & $46.62 \pm 6.89$ & $46.43 \pm 8.70$ & $47.25 \pm 8.70$ & 4.38 & 0.003 & {$[1]<[3]^{*}$} \\
\hline & & & & & & & \multicolumn{2}{|c|}{ (continued on next page) } \\
\hline
\end{tabular}




\begin{tabular}{|c|c|c|c|c|c|c|c|c|}
\hline Moral-religious emphasis (MRE) & $46.00 \pm 8.23$ & $47.80 \pm 10.83$ & $44.38 \pm 5.58$ & $47.21 \pm 5.41$ & $47.75 \pm 9.16$ & 0.45 & 0.769 & - \\
\hline Organisation (ORG) & $52.50 \pm 8.82$ & $52.40 \pm 10.54$ & $47.00 \pm 11.43$ & $48.14 \pm 10.47$ & $48.25 \pm 13.44$ & 1.24 & 0.301 & - \\
\hline Control (CTL) & $\begin{array}{l}47.93 \pm 8.10 \\
\text { Clinical } \\
\text { Variables }\end{array}$ & $46.48 \pm 8.30$ & $50.85 \pm 12.75$ & $52.29 \pm 9.34$ & $43.50 \pm 9.61$ & 1.73 & 0.150 & - \\
\hline PANSS positive & $7.53 \pm 1.08$ & $8.59 \pm 1.57$ & $12.68 \pm 3.68$ & $11.58 \pm 3.09$ & $18.00 \pm 5.65$ & 53.68 & $<0.001$ & $\begin{array}{l}{[1-2]<[3-5]^{* *}} \\
{[3-4]<[5]^{* *}}\end{array}$ \\
\hline PANSS negative & $9.39 \pm 3.07$ & $12.03 \pm 3.90$ & $17.53 \pm 5.40$ & $21.32 \pm 4.70$ & $21.95 \pm 3.52$ & 58.54 & $<0.001$ & $\begin{array}{l}{[1]<[2]^{*},} \\
{[1-2]<[3-5]^{* *},} \\
{[3]<[4-5]^{*}}\end{array}$ \\
\hline PANSS general & $18.06 \pm 2.13$ & $22.97 \pm 2.83$ & $28.53 \pm 4.33$ & $32.37 \pm 3.69$ & $42.20 \pm 5.39$ & 202.71 & $<0.001$ & $\begin{array}{l}{[1]<[2-5]^{* *},} \\
{[2]<[3-5]^{* *},} \\
{[3-4]<[5]^{* *},} \\
{[3]<[4]^{*}}\end{array}$ \\
\hline PANSS total & $34.98 \pm 4.90$ & $43.59 \pm 5.66$ & $58.74 \pm 8.15$ & $65.26 \pm 7.45$ & $82.15 \pm 10.37$ & 207.17 & $<0.001$ & $\begin{array}{l}{[1]<[2-5]^{* *},} \\
{[2]<[3-5]^{* *},} \\
{[3-4]<[5]^{* *},} \\
{[3]<[4]^{*}}\end{array}$ \\
\hline MADRS & $1.16 \pm 1.60$ & $4.59 \pm 3.90$ & $7.53 \pm 5.27$ & $10.95 \pm 4.54$ & $16.45 \pm 6.76$ & 56.23 & $<0.001$ & $\begin{array}{l}{[1]<[2]^{*},} \\
{[1]<[3-5]^{* *},} \\
{[2]<[4-5]^{* *},} \\
{[3]<[5]^{* *},} \\
{[4]<[5]^{*}}\end{array}$ \\
\hline $\begin{array}{l}\text { CGI } \\
\text { Psychosocial functioning }\end{array}$ & $1.80 \pm 1.08$ & $2.30 \pm 1.08$ & $3.53 \pm 1.12$ & $3.79 \pm 0.86$ & $4.15 \pm 1.18$ & 26.77 & $<0.001$ & {$[1-2]<[3-5]^{* *}$} \\
\hline Autonomy & $1.29 \pm 2.08$ & $2.08 \pm 2.11$ & $4.61 \pm 2.85$ & $4.95 \pm 3.22$ & $5.32 \pm 2.16$ & 17.21 & $<0.001$ & {$[1-2]<[3-5]^{* *}$} \\
\hline Occupational & $3.46 \pm 4.83$ & $4.08 \pm 4.55$ & $8.17 \pm 5.03$ & $7.32 \pm 4.16$ & $10.11 \pm 3.33$ & 10.56 & $<0.001$ & $\begin{array}{l}{[1-2]<[3-4]^{*}} \\
{[1-2]<[5]^{* *}}\end{array}$ \\
\hline Cognitive & $1.33 \pm 2.36$ & $3.24 \pm 3.28$ & $5.28 \pm 3.25$ & $6.84 \pm 2.67$ & $6.68 \pm 2.91$ & 20.73 & $<0.001$ & $\begin{array}{l}{[1]<[2]^{*}} \\
{[1]<[3]^{* *}} \\
{[1-2]<[4-5]^{* *}}\end{array}$ \\
\hline Financial & $0.31 \pm 1.11$ & $0.65 \pm 1.25$ & $1.17 \pm 1.82$ & $2.05 \pm 1.65$ & $2.11 \pm 1.56$ & 9.27 & $<0.001$ & $\begin{array}{l}{[1]<[4-5]^{* *}} \\
{[2]<[4-5]^{*}}\end{array}$ \\
\hline Interpersonal relationships & $2.21 \pm 3.40$ & $3.38 \pm 2.53$ & $6.61 \pm 3.82$ & $8.58 \pm 2.69$ & $8.95 \pm 3.37$ & 26.42 & $<0.001$ & $\begin{array}{l}{[1]<[4-5]^{* *},} \\
{[2]<[4-5]^{*}}\end{array}$ \\
\hline Leisure & $1.06 \pm 1.71$ & $1.41 \pm 1.26$ & $2.44 \pm 1.25$ & $2.84 \pm 1.68$ & $3.16 \pm 1.34$ & 10.61 & $<0.001$ & $\begin{array}{l}{[1]<[3-5]^{* *},} \\
{[2]<[3]^{*},} \\
{[2]<[3]^{*}} \\
{[2]<[4-5]^{* *}}\end{array}$ \\
\hline FAST total & $9.67 \pm 10.87$ & $14.84 \pm 10.07$ & $28.28 \pm 10.97$ & $32.58 \pm 11.22$ & $36.32 \pm 10.17$ & 34.06 & $<0.001$ & $\begin{array}{l}{[1]<[3]^{*},} \\
{[1]<[4-5]^{* *},} \\
{[2]<[4-5]^{*}}\end{array}$ \\
\hline
\end{tabular}

Abbreviations: $\mathrm{HSD}=$ Honestly Significant Difference; $M=$ Mean; $\mathrm{SD}=$ Standard Deviation; $\mathrm{SES}=$ Socioeconomic status; $\mathrm{DUP}=$ Duration of untreated psychosis; $\mathrm{CPZ}=\mathrm{Chlorpromazine} \mathrm{equiv-}$ alents; PAS= Premorbid Adjustment Scale; PANSS= Positive and Negative Symptom Scale; MADRS= Montgomery-Asberg Depression Rating Scale; CGI=Clinical Global Impression Scale;

FAST =Functioning Assessment Short Test. Significant differences $(p<0.05)$ marked in bold. ${ }^{*} p<0.05,{ }^{* *} p<0.001$. 
Table 3 Sociodemographic and clinical characteristics and psychosocial functioning among clinical trajectories at baseline and 2-year follow-up.

\begin{tabular}{|c|c|c|c|c|c|c|c|}
\hline & $\begin{array}{l}\text { Excellent } \\
\text { prognosis }(n=40) \\
{[1]}\end{array}$ & $\begin{array}{l}\text { Remitting course } \\
(n=46)[2]\end{array}$ & $\begin{array}{l}\text { Clinical worsening } \\
(n=11)[3]\end{array}$ & $\begin{array}{l}\text { Chronic course } \\
(n=28)[4]\end{array}$ & $\mathrm{F}$ & $p$ & $\begin{array}{l}\text { Post hoc } \\
\text { (Tukey's HSD) }\end{array}$ \\
\hline \multicolumn{8}{|l|}{ Sociodemographic variables } \\
\hline Gender: Male N(\%) & $29(73)$ & $30(65)$ & $5(46)$ & $20(72)$ & $\chi^{2}=3.18$ & 0.365 & - \\
\hline Age $(M \pm S D)$ & $24.98 \pm 5.19$ & $25.85 \pm 4.84$ & $25.82 \pm 4.38$ & $26.07 \pm 5.72$ & 0.32 & 0.812 & - \\
\hline \multirow[t]{6}{*}{ SES (\%) } & High & $9(23)$ & $12(26)$ & $4(36)$ & $3(11)$ & $\chi^{2}=17.80$ & $0.273-$ \\
\hline & Medium-High & $4(10)$ & $4(9)$ & $1(9)$ & $2(7)$ & & \\
\hline & Medium & $12(30)$ & $11(24)$ & $3(27)$ & $3(11)$ & & \\
\hline & Medium-Low & $8(20)$ & $16(35)$ & $3(27)$ & $16(57)$ & & \\
\hline & Low & $6(15)$ & $3(7)$ & $0(0)$ & $4(14)$ & & \\
\hline & Missing & $1(3)$ & $0(0)$ & $0(0)$ & $0(0)$ & & \\
\hline DUP & $75.06 \pm 81.95$ & $96.40 \pm 99.22$ & $70.90 \pm 93.72$ & $110.78 \pm 121.64$ & 0.83 & 0.480 & - \\
\hline Age of onset & $24.67 \pm 5.39$ & $25.49 \pm 4.76$ & $25.82 \pm 4.64$ & $25.61 \pm 5.41$ & 0.29 & 0.832 & - \\
\hline CPZ & $482.78 \pm 313.31$ & $720.44 \pm 408.12$ & $281.39 \pm 199.58$ & $710.31 \pm 397.94$ & 6.62 & $<0.001$ & $\begin{array}{l}{[1,3]<[2]^{*}} \\
{[3]<[4]^{*}}\end{array}$ \\
\hline CPZ FUP & $172.64 \pm 219.45$ & $161.64 \pm 240.07$ & $180.00 \pm 293.94$ & $252.53 \pm 299.46$ & 0.82 & 0.483 & - \\
\hline Alcohol: Yes N (\%) & $22(55)$ & $31(67)$ & $7(64)$ & $15(54)$ & $\chi^{2}=2.01$ & 0.571 & - \\
\hline Monthly alcohol FUP & $20.65 \pm 30.13$ & $50.83 \pm 88.09$ & $48.09 \pm 14.50$ & $29.54 \pm 62.76$ & $\chi^{2}=1.692$ & 0.172 & - \\
\hline Alcohol FUP & $24(60)$ & $29(63)$ & $7(64)$ & $13(47)$ & $\chi^{2}=4.23$ & 0.646 & - \\
\hline Monthly alcohol FUP & $17.48 \pm 29.07$ & $21.36 \pm 38.71$ & $30.11 \pm 45.75$ & $22.29 \pm 43.84$ & $\chi^{2}=0.739$ & 0.531 & - \\
\hline Cannabis: Yes N (\%) & $17(43)$ & $23(50)$ & $4(36)$ & $15(54)$ & $\chi^{2}=1.47$ & 0.688 & - \\
\hline Monthly cannabis & $38.15 \pm 58.23$ & $34.91 \pm 86.49$ & $28.09 \pm 53.40$ & $74.93 \pm 146.30$ & $\chi^{2}=1.784$ & 0.154 & - \\
\hline Cannabis FUP & $5(13)$ & $8(17)$ & $3(27)$ & $4(14)$ & $\chi^{2}=3.30$ & 0.770 & - \\
\hline Monthly cannabis FUP & $7.78 \pm 25.33$ & $21.73 \pm 42.35$ & $11.41 \pm 33.57$ & $16.07 \pm 42.01$ & $\chi^{2}=0.636$ & 0.593 & - \\
\hline Trauma: Yes N (\%) & $24(60)$ & $23(50)$ & $6(55)$ & $12(43)$ & $\chi^{2}=4.58$ & 0.598 & - \\
\hline PAS & $37.34 \pm 21.55$ & $42.44 \pm 16.57$ & $38.40 \pm 13.04$ & $56.50 \pm 24.14$ & 5.31 & 0.002 & {$[1]<[4]^{*}$} \\
\hline Cognitive reserve & $75.56 \pm 12.87$ & $77.47 \pm 9.58$ & $73.92 \pm 12.87$ & $71.73 \pm 12.01$ & 1.31 & 0.274 & - \\
\hline \multicolumn{8}{|l|}{ Family } \\
\hline History: Yes N (\%) & $6(15)$ & $11(24)$ & $6(55)$ & $7(25)$ & $\chi^{2}=7.42$ & 0.060 & - \\
\hline Cohesion $(C)$ & $50.08 \pm 6.81$ & $49.50 \pm 8.10$ & $49.20 \pm 8.83$ & $46.59 \pm 11.42$ & 0.94 & 0.425 & - \\
\hline C FUP & $52.49 \pm 7.74$ & $54.33 \pm 9.16$ & $52.50 \pm 10.95$ & $47.19 \pm 10.14$ & 2.27 & 0.087 & - \\
\hline Expressivity (EX) & $50.66 \pm 9.41$ & $51.00 \pm 8.23$ & $48.50 \pm 11.58$ & $45.89 \pm 10.56$ & 1.88 & 0.137 & - \\
\hline EXP FUP & $52.74 \pm 8.41$ & $54.57 \pm 7.68$ & $50.17 \pm 12.09$ & $46.63 \pm 8.42$ & 3.27 & 0.025 & {$[2]>[4]^{*}$} \\
\hline Conflicts (CON) & $48.11 \pm 7.03$ & $49.45 \pm 7.35$ & $48.00 \pm 6.88$ & $53.63 \pm 10.08$ & 2.87 & 0.039 & {$[1]<[4]^{*}$} \\
\hline CON FUP & $47.26 \pm 5.49$ & $47.47 \pm 4.26$ & $50.00 \pm 7.85$ & $46.50 \pm 5.73$ & 0.64 & 0.592 & - \\
\hline Independence (IND) & $49.66 \pm 8.59$ & $50.40 \pm 8.65$ & $49.60 \pm 9.45$ & $49.52 \pm 9.30$ & 0.08 & 0.973 & - \\
\hline IND FUP & $53.03 \pm 9.44$ & $54.50 \pm 7.15$ & $50.00 \pm 13.04$ & $50.88 \pm 8.43$ & 0.83 & 0.480 & - \\
\hline Achievement orientation (AO) & $47.39 \pm 9.09$ & $48.52 \pm 8.62$ & $41.00 \pm 15.28$ & $48.33 \pm 8.87$ & 1.78 & 0.154 & - \\
\hline AO FUP & $47.11 \pm 9.07$ & $47.13 \pm 9.09$ & $41.33 \pm 12.74$ & $45.50 \pm 10.09$ & 0.74 & 0.532 & - \\
\hline Intellectual-cultural orientation (ICO) & $46.82 \pm 10.19$ & $49.60 \pm 10.64$ & $48.90 \pm 8.69$ & $44.70 \pm 10.09$ & 1.38 & 0.252 & - \\
\hline
\end{tabular}


Table 3 (continued)

\begin{tabular}{|c|c|c|c|c|c|c|c|}
\hline ICO FUP & $50.06 \pm 10.74$ & $54.17 \pm 12.36$ & $51.83 \pm 10.07$ & $45.06 \pm 11.36$ & 2.28 & 0.085 & - \\
\hline Active-recreational orientation (ARO) & $52.03 \pm 7.78$ & $49.50 \pm 8.52$ & $49.80 \pm 8.22$ & $45.48 \pm 6.42$ & 3.71 & 0.014 & {$[1]>[4]^{*}$} \\
\hline ARO FUP & $55.86 \pm 8.50$ & $52.93 \pm 10.57$ & $46.83 \pm 10.72$ & $46.69 \pm 7.92$ & 4.35 & 0.007 & {$[1]>[4]^{*}$} \\
\hline Moral-religious emphasis (MRE) & $46.50 \pm 7.78$ & $45.07 \pm 8.38$ & $46.00 \pm 8.88$ & $46.22 \pm 7.38$ & 0.24 & 0.871 & - \\
\hline MRE FUP & $45.71 \pm 7.57$ & $47.83 \pm 10.96$ & $49.83 \pm 6.65$ & $46.50 \pm 6.83$ & 0.56 & 0.640 & - \\
\hline Organisation (ORG) & $51.87 \pm 8.80$ & $48.76 \pm 10.18$ & $50.70 \pm 10.99$ & $48.37 \pm 11.16$ & 0.89 & 0.447 & - \\
\hline ORG FUP & $52.14 \pm 9.37$ & $52.83 \pm 9.66$ & $47.33 \pm 10.73$ & $48.50 \pm 11.85$ & 1.04 & 0.380 & - \\
\hline Control (CTL) & $48.71 \pm 8.30$ & $46.79 \pm 6.52$ & $43.80 \pm 8.64$ & $50.48 \pm 7.99$ & 2.41 & 0.071 & - \\
\hline CTL FUP & $47.54 \pm 8.73$ & $47.17 \pm 7.54$ & $46.00 \pm 11.31$ & $50.25 \pm 9.84$ & 0.57 & 0.638 & - \\
\hline \multicolumn{8}{|l|}{ Clinical Variables } \\
\hline PANSS positive & $10.73 \pm 4.52$ & $23.39 \pm 6.97$ & $12.00 \pm 5.83$ & $21.86 \pm 6.70$ & 37.97 & $<0.001$ & {$[1,3]<[2,4]^{* *}$} \\
\hline PANSS positive FUP & $7.93 \pm 1.29$ & $8.04 \pm 1.52$ & $16.36 \pm 7.97$ & $14.29 \pm 4.35$ & 40.05 & $<0.001$ & {$[1-2]<[3-4]^{* *}$} \\
\hline PANSS negative & $13.58 \pm 5.45$ & $22.17 \pm 6.52$ & $11.36 \pm 5.14$ & $23.25 \pm 6.89$ & 24.27 & $<0.001$ & {$[1,3]<[2,4]^{* *}$} \\
\hline PANSS negative FUP & $10.35 \pm 3.63$ & $10.67 \pm 3.81$ & $20.36 \pm 3.98$ & $22.14 \pm 4.09$ & 75.80 & $<0.001$ & {$[1-2]<[3-4]^{* *}$} \\
\hline PANSS general & $24.55 \pm 4.76$ & $45.67 \pm 8.69$ & $25.91 \pm 4.21$ & $45.61 \pm 8.89$ & 79.99 & $<0.001$ & {$[1,3]<[2,4]^{* *}$} \\
\hline PANSS general FUP & $19.73 \pm 3.48$ & $20.57 \pm 3.42$ & $38.18 \pm 6.16$ & $37.11 \pm 7.07$ & 118.23 & $<0.001$ & {$[1-2]<[3-4]^{* *}$} \\
\hline PANSS total & $48.85 \pm 10.47$ & $91.24 \pm 14.38$ & $49.27 \pm 9.07$ & $90.71 \pm 14.99$ & 105.45 & $<0.001$ & {$[1,3]<[2,4]^{* *}$} \\
\hline PANSS total FUP & $38.00 \pm 6.84$ & $39.28 \pm 6.68$ & $74.91 \pm 15.88$ & $73.54 \pm 11.03$ & 139.58 & $<0.001$ & {$[1-2]<[3-4]^{* *}$} \\
\hline MADRS & $5.25 \pm 5.28$ & $15.43 \pm 9.89$ & $9.00 \pm 5.74$ & $16.14 \pm 8.48$ & 15.28 & $<0.001$ & {$[1]<[2,4]^{* *}$} \\
\hline MADRS FUP & $1.98 \pm 2.81$ & $3.22 \pm 3.59$ & $17.82 \pm 7.86$ & $12.18 \pm 4.96$ & 67.10 & $<0.001$ & $\begin{array}{l}{[1-2]<[3-4]^{* *},} \\
{[3]>[4]^{*}}\end{array}$ \\
\hline CGI & $3.93 \pm 1.07$ & $4.59 \pm 1.13$ & $3.64 \pm 1.21$ & $4.32 \pm 0.82$ & 4.10 & 0.008 & {$[1,3]<[2]^{*}$} \\
\hline CGI FUP & $1.98 \pm 1.12$ & $2.04 \pm 1.09$ & $4.45 \pm 0.69$ & $3.79 \pm 1.10$ & 30.75 & $<0.001$ & {$[1-2]<[2-4]^{* *}$} \\
\hline \multicolumn{8}{|l|}{ Psychosocial functioning } \\
\hline Autonomy & $3.10 \pm 3.23$ & $5.04 \pm 3.62$ & $2.27 \pm 2.41$ & $5.22 \pm 3.25$ & 4.47 & 0.005 & {$[1]<[2]^{*}$} \\
\hline Autonomy FUP & $1.51 \pm 2.04$ & $1.74 \pm 2.21$ & $5.10 \pm 3.87$ & $5.14 \pm 2.26$ & 19.69 & $<0.001$ & {$[1-2]<[3-4]^{* *}$} \\
\hline Occupational & $7.15 \pm 6.05$ & $10.43 \pm 4.71$ & $4.27 \pm 4.71$ & $8.70 \pm 3.80$ & 5.84 & 0.001 & \\
\hline Occupational FUP & $3.13 \pm 4.88$ & $4.24 \pm 4.53$ & $8.90 \pm 3.90$ & $8.64 \pm 4.08$ & 11.15 & $<0.001$ & {$[1-2]<[3-4]^{* *}$} \\
\hline Cognitive & $3.90 \pm 3.24$ & $7.13 \pm 3.77$ & $3.00 \pm 2.00$ & $7.37 \pm 3.05$ & 11.27 & $<0.001$ & {$[1,3]<[2]^{*}$} \\
\hline Cognitive FUP & $1.79 \pm 2.70$ & $2.48 \pm 3.13$ & $6.90 \pm 3.45$ & $6.71 \pm 2.54$ & 22.51 & $<0.001$ & {$[1-2]<[3-4]^{* *}$} \\
\hline Financial & $0.67 \pm 1.51$ & $2.04 \pm 2.04$ & $1.00 \pm 1.84$ & $2.07 \pm 1.41$ & 5.75 & 0.001 & {$[1,3]<[2,4]^{* *}$} \\
\hline Financial FUP & $0.28 \pm 0.86$ & $0.61 \pm 1.39$ & $2.70 \pm 1.89$ & $1.86 \pm 1.43$ & 14.98 & $<0.001$ & {$[1-2]<[3-4]^{* *}$} \\
\hline Interpersonal relationships & $3.97 \pm 4.18$ & $8.80 \pm 4.53$ & $3.27 \pm 3.47$ & $9.33 \pm 4.51$ & 14.30 & $<0.001$ & {$[1]<[2,4]^{*}$} \\
\hline Interpersonal relationships FUP & $1.82 \pm 2.17$ & $3.48 \pm 3.54$ & $8.40 \pm 2.95$ & $8.89 \pm 3.08$ & 37.51 & $<0.001$ & {$[1-2]<[3-4]^{* *}$} \\
\hline Leisure & $1.46 \pm 1.60$ & $2.54 \pm 1.94$ & $1.36 \pm 1.50$ & $2.85 \pm 1.79$ & 4.86 & 0.003 & {$[1,3]<[2,4]^{* *}$} \\
\hline Leisure FUP & $1.03 \pm 1.35$ & $1.37 \pm 1.66$ & $3.00 \pm 1.63$ & $3.00 \pm 1.49$ & 12.35 & $<0.001$ & {$[1-2]<[3-4]^{* *}$} \\
\hline FAST & $20.26 \pm 13.83$ & $36.00 \pm 14.88$ & $15.18 \pm 10.78$ & $35.56 \pm 13.49$ & 14.57 & $<0.001$ & {$[1]<[2,4]^{*}$} \\
\hline FAST FUP & $9.56 \pm 9.18$ & $13.91 \pm 11.70$ & $35.00 \pm 14.23$ & $34.25 \pm 9.49$ & 39.99 & $<0.001$ & {$[1-2]<[3-4]^{* *}$} \\
\hline
\end{tabular}

Abbreviations: $\mathrm{HSD}=$ Honestly Significant Difference; $M=$ Mean; $\mathrm{SD}=$ Standard Deviation; $\mathrm{PC}=$ Principal Component; $\mathrm{SES}=$ Socioeconomic status; $\mathrm{DUP}=\mathrm{Duration}$ of untreated psychosis; $\mathrm{CPZ}=$ Chlorpromazine equivalents; PAS= Premorbid Adjustment Scale; FUP = Follow-up; PANSS= Positive and Negative Symptom Scale; MADRS= Montgomery-Asberg Depression Rating Scale; $\mathrm{CGI}=$ Clinical Global Impression Scale; FAST=Functioning Assessment Short Test. Significant differences $(p<0.05)$ marked in bold. ${ }^{*} p<0.05,{ }^{* *} p<0.001$. 


\section{Discussion}

As far as we are aware, this is the first study to use a machine learning algorithms method to identify individual clinical trajectories of FEP patients and their risk factors based on their psychopathological presentation at baseline and 2-year follow-up. Three main findings emerged from the present study: 1) PCA of the PANSS and MADRS yielded six symptom factors (positive, negative, depressive, anxiety, disorganisation and somatic/cognitive) at baseline and 2-year follow-up; 2) There are three well defined clinical clusters at baseline ("mild", "negative and moderate" and "positive and severe") and five at follow-up ("minimal", "mild", "moderate", "negative and depressive" and "severe symptoms"); 3) The risk factors contributing towards a clinical worsening in patients with non-affective FEP were low doses of antipsychotics, high depressive symptomatology and the presence of a positive family history whilst the protective factors for a remitting course were a high CR and better premorbid adjustment.

The six-factor model included 36 items at baseline (26 PANSS items and 10 MADRS items) and 34 items at followup (25 PANSS items and 9 MADRS items). The majority of studies on the PANSS agreed that either four or five dimensions describe the psychosis construct, with positive, negative, disorganisation, and affective symptom dimensions most frequently reported (Potuzak et al., 2012) and some studies reported an independent anxiety dimension (Cuesta et al., 2003; Allardyce et al., 2007). As depressive symptoms are common in patients with a FEP, with a reported prevalence from $7 \%$ to $75 \%$ in the different studies (Sim et al., 2004), our study also included one measure of depression (MADRS). Previous investigations of the MADRS structure have differed in research design (i.e. samples have included patients with a range of diagnoses) and analytic strategy, constraining comparisons across studies (Quilty et al., 2013). The number of factors identified in literature ranged from one-unidimensional (Lobo et al., 2002; Carmody et al., 2006); two (Rocca et al., 2002; Uher et al., 2008); three (Suzuki et al., 2005; Ketharanathan et al., 2015); and up to four dimensions (Williamson et al., 2006). Almost all studies with three or four factors found dimensions characterised by sadness/dysphoria/anhedonia, anxiety and vegetative/somatic symptoms. Thus, the factors identified in our study by the PCA of the PANSS and MADRS scales are essentially the same as those described in the majority of other studies, namely positive, negative, disorganised, depressive, anxiety and somatic. However, the components do not have exactly the same composition at both times of the evaluations. The instability of the PCA could express the role played by the phase of illness (Dollfus and Petit, 1995). For example, at baseline the positive component included excitement, conceptual disorganization, suspiciousness/persecution and lack of insight, but these items appeared in disorganization component at follow-up. This results can be due to the fact that after a first-episode, the positive symptoms tend to reduce, whereas follow-up studies have suggested that after antipsychotic medication stabilization, negative symptoms tend to be stable and persistent (Ventura et al., 2015; Mezquida et al., 2017).

The six factors described above were used to conduct a cluster analysis that allowed clinical subtypes of patients with FEP to be identified. Three distinct patient clusters have been determined at baseline: the first cluster was characterised by mild symptoms (39\%), a second cluster had negative and moderate symptoms (31\%) and a third presented positive and severe symptoms (30\%). Patients with negative symptoms in comparison with those presenting mild symptoms had higher DUP and doses of antipsychotics, worse premorbid adjustment, higher illness severity and lower psychosocial functioning. These results are in line with previous studies and confirm that a poor premorbid functioning is associated with worse psychosocial functioning (White et al., 2009; Wunderink et al., 2020), and with the severity of clinical presentation, especially for patients with a FEP presenting negative symptoms (Amoretti et al., 2016; Mezquida et al., 2017). Previous reports have also supported the association between negative symptoms and DUP (Perkins et al., 2005). At 2-year follow-up, five subtypes of patients were derived: "minimal”, “mild”, “moderate”, "negative and depressive" and "severe symptoms". Patients with minimal or mild symptoms had better premorbid adjustment, higher family cohesion and lower conflicts as well as more intellectual-cultural and active-recreational orientation, lower illness severity and greater functioning. An increasingly large number of studies have found an association between negative family environment and poor prognosis in FEP patients (Norman et al., 2005; Lee et al., 2013). Higher rates of negative family environmental styles are associated with an increased likelihood of relapse (Wuerker, 1996; Butzlaff and Hooley, 1998).

In the present article, four trajectories were identified, namely "excellent prognosis", "remitting course", "clinical worsening" and "chronic course". According to previous studies, a significant number of people achieving symptom remission and recovery were found (Van Os and Kapur, 2009; Austin et al., 2015) and a small but significant group also showed a deteriorating course (Rabinowitz et al., 2007). Results indicate that the majority of participants showed a general pattern of reduction of positive symptoms, in line with previous literature (Austin et al., 2015). However, previously it has also been reported that negative symptoms typically showed less variation during the ten years after the first episode (Austin et al., 2015). On the contrary, in our study negative symptoms showed an important reduction. These results could perhaps be explained by the difficulty in detecting secondary negative symptoms caused by positive symptoms, depression, and/or extrapyramidal symptoms amongst other factors, which could be present in our patients. These secondary symptoms represent a diagnostic challenge, particularly in the early stages after a first episode, when positive symptomatology predominates (Mezquida et al., 2017).

Patients who presented a clinical worsening were characterised by having lower CPZ and higher depressive symptoms at baseline, and more presence of family history. Depression is significant in the prediction of transition to FEP, poor clinical outcomes, quality of life, and suicide (FusarPoli et al., 2014; Gardsjord et al., 2016). Similarly, a positive psychiatric family history was found to be associated with worse outcome (Esterberg and Compton, 2012). Confirming previous studies, patients with a remitting course showed better premorbid adjustment and higher CR compared to those with a chronic course (Mezquida et al., 2017; 
Amoretti et al., 2016, 2018, 2020). Thus, these results suggest that prescribing higher doses of antipsychotics as well as addressing depressive symptomatology can help to prevent clinical worsening. Undoubtedly, there are risk factors that cannot be changed, such as the presence of a positive psychiatric family history. Nonetheless, more attention should be paid to these subgroup of FEP patients as they already present a higher risk of worse outcomes. Since a high CR and better premorbid adjustment represented the protective factors for a remitting course in our study, targeting older, less-educated, poor patients and their families seems necessary. Similarly, in the comparison between those patients who showed "minimal" or "mild" symptoms versus those who presented "negative and depressive" or "severe" symptoms at follow-up, differences emerged in terms of premorbid adjustment, family expression and active-recreational orientation. There were no differences between the four trajectories in terms of alcohol or cannabis use. Although cannabis use has been shown to increase the risk of developing psychosis and is related to a poor prognosis, our study did not find this association. This may be due to the fact that the age of initiation of cannabis use, the duration of cannabis use and cannabis patterns may also play an important role (Forti et al., 2014) and were not evaluated in this study.

As a consequence, the findings of the present study highlight that the symptom trajectories among patients with FEP are heterogeneous (Abdin et al., 2017) and should not be over-simplified. Psychopathology at the beginning of the episode must be taken into account to understand the trajectory of each person in an attempt to develop tailored intervention and personalised treatment strategies. For instance, different interventions should be applied to those patients presenting moderate symptoms at baseline and still suffering the same symptomatology at follow-up than that applied to those experiencing mild symptoms at baseline and ultimately presenting a worsening in the symptomatology. Current staging models are based on the integration of clinical baseline information, such as psychopathological presentation at the early stages of psychosis, with trajectories of the disease. Defining each patient's characteristics, severity, and prognosis in a more precise and individualised way might aid the clinical decision-making algorithm to decide which treatment is most effective at that specific stage on the basis of the patient's characteristics (Salagre et al., 2018).

Some limitations have to be taken into consideration before translating these findings into clinical practice. Firstly, a limitation of all CR studies evaluating psychiatric populations is that there was no validated instrument to measure CR so criteria established and replicated in previous studies were followed. Indeed, the Cognitive Reserve Assessment Scale in Health (CRASH) (Amoretti et al., 2019) has been validated in 2019. It is the first tool to assess CR in patients with severe mental illness. A second limitation is that no specific scale was used to assess negative symptomatology, due to constraints associated with the PANSS scale (Blanchard et al., 2011). Future studies making use of improved negative symptom scales- such as the Brief Negative Symptom Scale (BNSS) (Kirkpatrick et al., 2011) or the Clinical Assessment Interview for Negative Symptoms (CAINS) (Kring et al., 2013)-might differentiate between primary and sec- ondary negative symptoms (Potuzak et al., 2012; Mezquida et al., 2017), which is an unmet need of current research on FEP. Thirdly, the retrospective assessment of the premorbid phase. While retrospective reports from patients about their childhood and developmental adjustment may be subject to recall bias, it has been shown that premorbid adjustment measured by PAS scale is related to important clinical features of the illness (Rabinowitz et al., 2006: Austin et al., 2015; Amoretti et al., 2020). It is important to note that this is a naturalistic, multicentric and longitudinal study and that there was no other way to collect this premorbid information. Finally, despite few patients were prescribed with high doses of antipsychotics, according to a previous study by the same group assessing pharmacovigilance in the same sample of FEP patients, a total of $87.2 \%$ of the patients were receiving adequate doses of antipsychotic, according to the recommended dose range in the specifications of the product for Spain for the patient age (Bioque et al., 2016). Due to its naturalistic design and by the very nature of the sample included, some treatment prescription could be higher than dose recommended in prescribing guidelines. Nonetheless, these data provide a real-world point of view on the clinical prescription in FEP patients on a country basis.

In spite of its limitations, the strength of this study lies in the evaluation of FEP patients that represent quite an ideal population for exploring the clinical expression of the core symptoms of psychoses. The inclusion of a wellcharacterised and naturalistic sample of non-affective FEP, the longitudinal study design, the clinical assessment of both symptoms and functioning, and the comprehensive and sophisticated statistical assessment used are the strengths of the present work, reporting clinically sound results that can be implemented in daily clinical practice.

In conclusion, the adoption of a dimensional approach in FEP might represent an advantage in clinical practice by providing a more comprehensive description of the complexity of FEP psychopathology (Tonna et al., 2019). This approach might also allow for an improvement in the accuracy of targeting therapeutic interventions. Not only positive but also negative and depressive symptoms are common in patients with FEP and are highly involved in their clinical trajectory. Thus, this study highlights the importance of addressing depressive and negative symptoms, cognitive reserve and premorbid adjustment in the early stages of psychosis.

\section{Role of funding source}

The funding sources were not involved in the study design, conduct, monitoring and preparation of the final database and had no influence on the final data analysis of this report.

\section{Contributors}

SA and NV designed the study, managed the literature searches and analyses, undertook the statistical analysis, and wrote the first draft of the manuscript. GM, FDR, BS, $A C, M B$ and EV revised the first draft and added critical comments to guide the redaction of the final manuscript. MJC, LPC, MGR, CC, AGP, CDM, IC, ES, MA and AM revise the 
second draft of the article and provided critical comments to guide the redaction of the final manuscript. All the authors within the PEPs Group recluted patients and healthy controls at their centers, provided the anonimous data and revise the final manuscript. All authors approved the final manuscript.

\section{Conflict of interest}

E. Vieta has received research support from or served as consultant, adviser or speaker for AB-Biotics, Actavis, Allergan, Angelini, AstraZeneca, Bristol-Myers Squibb, Dainippon Sumitomo Pharma, Ferrer, Forest Research Institute, Gedeon Richter, Glaxo-Smith-Kline, Janssen, Lundbeck, Otsuka, Pfizer, Roche, Sanofi-Aventis, Servier, Shire, Sunovion, Takeda, Telefónica, the Brain and Behaviour Foundation, the Spanish Ministry of Science and Innovation (CIBERSAM), the Seventh European Framework Programme (ENBREC), and the Stanley Medical Research Institute, unrelated to the present work.

M. Bernardo has been a consultant for, received grant/research support and honoraria from, and been on the speakers/advisory board of ABBiotics, Adamed, Angelini, Casen Recordati, Janssen-Cilag, Lundbeck, Otsuka, Menarini and Takeda.

C. De-la-Camara received financial support to attend scientific meetings from Janssen-Cilag, Almirall, Eli Lilly, Lundbeck, Rovi, Esteve, Novartis, and Astrazeneca

M. Gómez-Ramiro reports grants from INSTITUTO CARLOS III, grants from ADAMED, non-financial support from JANSSEN-CILAG, non-financial support from LUNDBECKOTSUKA, non-financial support from PFIZER, non-financial support from ANGELINI, outside the submitted work.

R. Rodriguez-Jimenez has been a consultant for, spoken in activities of, or received grants from: Instituto de Salud Carlos III, Fondo de Investigación Sanitaria (FIS), Centro de Investigación Biomédica en Red de Salud Mental (CIBERSAM), Madrid Regional Government (S2010/ BMD-2422 AGES; S2017/BMD-3740), JanssenCilag, Lundbeck, Otsuka, Pfizer, Ferrer, Juste, Takeda, Exeltis, Casen-Recordati, Angelini.

The rest of authors report no biomedical financial interests or potential conflicts of interest.

\section{Acknowledgments}

We are extremely grateful to all participants.

This study is part of a coordinated-multicentre Project, funded by the Ministerio de Economía y Competitividad (PI08/0208; PI11/00325; PI14/00612), Instituto de Salud Carlos III - Fondo Europeo de Desarrollo Regional. Unión Europea. Una manera de hacer Europa, Centro de Investigación Biomédica en Red de salud Mental, CIBERSAM, by the CERCA Programme / Generalitat de Catalunya AND Secretaria d'Universitats i Recerca del Departament d'Economia I Coneixement (2017SGR1355). Departament de Salut de la Generalitat de Catalunya, en la convocatoria corresponent a l'any 2017 de concessió de subvencions del Pla Estratègic de Recerca i Innovació en Salut (PERIS) 2016-2020, modalitat Projectes de recerca orientats a l'atenció primària, amb el codi d'expedient SLT006/17/00345.
S. Amoretti has been supported by a Sara Borrell (CD20/00177), funded by Instituto de Salud Carlos III (ISCIII) and co-funded by European Social Fund "Investing in your future".

The study has been supported by a BITRECS project conceded to N. Verdolini. BITRECS project has received funding from the European Union's Horizon 2020 research and innovation programme under the Marie Skłodowska-Curie grant agreement No 754550 and from "La Caixa" Foundation (ID 100010434), under the agreement LCF/PR/GN18/50310006.

E. Vieta thanks the support of the Spanish Ministry of Science, Innovation and Universities (PI18/00805) integrated into the Plan Nacional de $I+D+I$ y cofinanciado por el ISCIII-Subdirección General de Evaluación y el Fondo Europeo de Desarrollo Regional (FEDER); the Instituto de Salud Carlos III; the CIBER of Mental Health (CIBERSAM); the Secretaria d'Universitats i Recerca del Departament d'Economia i Coneixement (2017 SGR 1365) and the CERCA Programme / Generalitat de Catalunya) and the CERCA Programme / Generalitat de Catalunya. We would like to thank the Departament de Salut de la Generalitat de Catalunya for the PERIS grant SLT006/17/00357.

Finally, we would also like to thank the authors of the PEPs group who participated in the development of this manuscript, namely, Bioque $M^{1,2}$, Pons $M T{ }^{1}$, AndreuBernabeu Á ${ }^{2,7}$, Ayora $M^{2,7}$, Alonso-Solís A ${ }^{2,10}$, Grasa E 2,10, González-Ortega I 2,9,15, Zorrilla I 2,9, Saz P 8 , Santabárbara $\mathrm{J}^{2,16}$, Nacher J 2,17, Castro-Bleda MJ ${ }^{18}$, Toll A ${ }^{2,12}$, Martinez L ${ }^{19}$, Sanchez-Moreno J 2,3, Anmella G 1, Castro-Fornieles J 2,11, Baeza | 2,11, Contreras F 2,20, Saiz-Masvidal C 20,21, García Álvarez L 2,22,23, Bobes-Bascarán T 2,23,24, Gutiérrez M 2,25 , Segarra $R{ }^{2,26}$, Sanchez-Pastor $L{ }^{27}$, Rodriguez-Jimenez $\mathrm{R}^{2,27,28}$, Usall J ${ }^{29}$, Butjosa A ${ }^{29}$, Sarró S ${ }^{30}$, Pomarol-Clotet $E$ ${ }^{30}$, Ibáñez $A^{2,31}$, Sánchez-Torres $A M^{5,6}$, Balanzá-Martinez $V$ 2,32 .

PEPs Group additional affiliations:

${ }^{15}$ The National Distance Education University (UNED), Vitoria, Spain.

16 Department of Microbiology, Preventive Medicine and Public Health, Universidad de Zaragoza, Zaragoza, Spain.

17 Neurobiology Unit, Program in Neurosciences and Interdisciplinary Research Structure for Biotechnology and Biomedicine (BIOTECMED), Universitat de València, Biomedical Research Institute INCLIVA, Valencia, Spain.

18 VRAIN: Valencian Research Institute for Artificial Intelligence, Universitat Politècnica de València, Valencia, Spain.

${ }^{19}$ Hospital del Mar, Department of Psychiatry, Barcelona, Spain.

20 Bellvitge Biomedical Research Institute IDIBELL, Department of Psychiatry- Bellvitge University Hospital, Hospitalet de Llobregat- Barcelona, Spain.

21 University of Barcelona, Department of Clinical Sciences- School of Medicine, Barcelona, Spain.

22 Department of Psychiatry, Universidad de Oviedo, Oviedo, Spain.

${ }^{23}$ Servicio de Salud del Principado de Asturias (SESPA) Oviedo, Instituto de Investigación Sanitaria del Principado de Asturias (ISPA), Instituto Universitario de Neurociencias del Principado de Asturias (INEUROPA), Oviedo, Spain.

24 Department of Psycology, Universidad de Oviedo, Oviedo, Spain 


\author{
${ }^{25}$ Araba University Hospital, Bioaraba Research Institute, \\ Vitoria, Spain. \\ ${ }^{26}$ Cruces University Hospital, BioCruces Health Research \\ Institute, University of the Basque Country (UPV/EHU), Viz- \\ caya, Spain. \\ 27 Instituto de Investigación Sanitaria Hospital 12 de Oc- \\ tubre (imas12), Madrid, Spain. \\ 28 CogPsy Group, Universidad Complutense de Madrid \\ (UCM), Madrid, Spain. \\ ${ }^{29}$ Institut de Recerca Sant Joan de Déu, Esplugues del \\ Llobregat, Parc Sanitari Sant Joan de Déu, Sant Boi de Llo- \\ bregat, Barcelona, Spain. \\ 30 FIDMAG Germanes Hospitalàries Research Foundation, \\ Barcelona, Spain. \\ 31 Department of Psychiatry, Hospital Universitario Ramón \\ y Cajal, IRYCIS, Universidad de Alcalá, Madrid, Spain. \\ 32 Department of Medicine, University of Valencia, Valen- \\ cia, Spain.
}

\section{Supplementary materials}

Supplementary material associated with this article can be found, in the online version, at doi:10.1016/j.euroneuro. 2021.01.095.

\section{References}

Abdin, E., Chong, S.A., Vaingankar, J.A., Peh, C.X., Poon, L.Y., Rao, S., Verma, S., Subramaniam, M., 2017. Trajectories of positive, negative and general psychopathology symptoms in first episode psychosis and their relationship with functioning over a 2-year follow-up period. PLoS One 12, e0187141.

Allardyce, J., McCreadie, R.G., Morrison, G., van Os, J., 2007. Do symptom dimensions or categorical diagnoses best discriminate between known risk factors for psychosis? Soc. Psychiatry Psychiatr. Epidemiol. 42, 429-437.

Allott, K., Liu, P., Proffitt, T.M., Killackey, E., 2011. Cognition at illness onset as a predictor of later functional outcome in early psychosis: systematic review and methodological critique. Schizophr. Res. 125 (2-3), 221-235.

American Psychiatric Association, 2013. Diagnostic and Statistical Manual of Mental Disorders (DSM-5 ${ }^{\circledR}$ ), 5th edn. Arlington: American Psychiatric Publishing, Arlington.

Amoretti, S., Bernardo, M., Bonnin, C.M., Bioque, M., Cabrera, B., Mezquida, G., Solé, B., Vieta, E., Torrent, C., 2016. The impact of cognitive reserve in the outcome of first-episode psychoses: 2-year follow-up study. Eur. Neuropsychopharmacol. 26, 1638-1648.

Amoretti, S., Cabrera, B., Torrent, C., Mezquida, G., Lobo, A., González-Pinto, A., Parellada, M., Corripio, I., Vieta, E., de la Serna, E., Butjosa, A., Contreras, F., Sarró, S., Penadés, R., Sánchez-Torres, A.M., Cuesta, M., Bernardo, M., 2018. Cognitive reserve as an outcome predictor: first-episode affective versus non-affective psychosis. Acta Psychiatr. Scand. 138, 411-455.

Amoretti, S., Cabrera, B., Torrent, C., Bonnín, C.M., Mezquida, G., Garriga, M., Jiménez, E., Martínez-Arán, A., Solé, B., Reinares, M., Varo, C., Penadés, R., Grande, I., Salagre, E., Parellada, E., Bioque, M., Garcia-Rizo, C., Meseguer, A., Anmella, G., Rosa, A.R., Contreras, F., Safont, G., Vieta, E., Bernardo, M., 2019. Cognitive reserve assessment scale in health (CRASH): its validity and reliability. J Clin. Med. 28, 8 .

Amoretti, S., Rosa, A.R., Mezquida, G., Cabrera, B., Ribeiro, M., Molina, M., Bioque, M., Lobo, A., González-Pinto, A.,
Fraguas, D., Corripio, I., Vieta, E., de la Serna, E., Morro, L., Garriga, M., Torrent, C., Cuesta, M.J., Bernardo, M., 2020. The impact of cognitive reserve, cognition and clinical symptoms on psychosocial functioning in first-episode psychoses. Psychol. Med. 1-12.

Austin, S.F., Mors, O., Budtz-Jorgensen, E., Secher, R.G., Hjorthoj, C.R., Bertelsen, M., Jeppesen, P., Petersen, L., Thorup, A., Nordentoft, M., 2015. Long-term trajectories of positive and negative symptoms in first episode psychosis: a 10 year follow-up study in the OPUS cohort. Schizophr. Res. 168 (1-2), 84-91.

Barnett, J., Salmond, C., Jones, P., Sahakian, B.J., 2006. Cognitive reserve in neuropsychiatry. Psychol. Med. 36, 1053-1064.

Bioque, M., Llerena, A., Cabrera, B., Mezquida, G., Lobo, A., González-Pinto, A., Díaz-Caneja, C.M., Corripio, I., Aguilar, E.J., Bulbena, A., Castro-Fornieles, J., Vieta, E., Lafuente, A., Mas, S., Parellada, M., Saiz-Ruiz, J., Cuesta, M.J., Bernardo, M., 2016. A Pharmacovigilance study in first episode of psychosis: psychopharmacological interventions and safety profiles in the PEPs project. Int. J. Neuropsychopharmacol. 19 (4), pyv121.

Bernardo, M., Bioque, M., Parellada, M., Saiz, J., Cuesta, M.J., Llerena, A., Sanjuán, J., Castro-Fornieles, J., Arango, C., Cabrera, B., 2013. Assessing clinical and functional outcomes in a gene-environment interaction study in first episode of psychosis (PEPs). Rev. Psiquiatr. Salud Ment. 6, 4-16.

Bernardo, M., Cabrera, B., Arango, C., Bioque, M., CastroFornieles, J., Cuesta, M.J., Lafuente, A., Parellada., M., Saiz-Ruiz, J., Vieta, E, 2019. One decade of the first episodes project (PEPs): advancing towards a precision psychiatry. Rev. Psiquiatr. Salud Ment. 12, 135-140.

Blanchard, J.J., Kring, A.M., Horan, W.P., Gur, R., 2011. Toward the next generation of negative symptom assessments: the collaboration to advance negative symptom assessment in schizophrenia. Schizophr. Bull. 37, 291-299.

Butzlaff, R.L., Hooley, J.M., 1998. Expressed emotion and psychiatric relapse: a meta-analysis. Arch. Gen. Psychiatry 55 (6), 547-552.

Cannon-Spoor, H., Potkin, S., Wyatt, R., 1982. Measurement of premorbid adjustment in chronic schizophrenia. Schizophr. Bull. 8, 470-484.

Carmody, T.J., Rush, A.J., Bernstein, I., Warden, D., Brannan, S., Burnham, D., Woo, A., Trivedi, M.H., 2006. The Montgomery Äsberg and the Hamilton ratings of depression: a comparison of measures. Eur. Neuropsychopharmacol. 16 (8), 601-611.

Cocchi, A., Cerati, G., Lora, A., Meneghelli, A., Monzani, E., Percudani, M., Petrovich, L., Mirabella, F., Picardi, A., Preti, A., 2014. Patients with first-episode psychosis are not a homogeneous population: implications for treatment. Clin. Pract. Epidemiol. Ment. Health 10, 1-8.

Colasanti, A., Paletta, S., Moliterno, D., Mazzocchi, A., Mauri, M.C., Altamura, A.C., 2010. Symptom dimensions as predictors of clinical outcome, duration of hospitalization and aggressive behaviours in acutely hospitalized patients with psychotic exacerbation. Clin. Pract. Epidemiol. Ment. Health 6, 72-78.

Compton, M.T., Kelley, M.E., Ionescu, D.F., 2014. Subtyping first-episode non-affective psychosis using four early-course features: potentially useful prognostic information at initial presentation. Early Interv. Psychiatry 8 (1), 50-58.

Cuesta, M.J., Peralta, V., Gil, P., Artamendi, M., 2003. Psychopathological dimensions in first-episode psychoses. From the trunk to the branches and leaves. Eur. Arch. Psychiatry Clin. Neurosci. 253, 73-79.

Davidson, J., Smith, R., 1990. Traumatic experiences in psychiatric outpatients. J. Trauma Stress 3, 459-475.

De la Serna, E., Andrés-Perpiñá, S., Puig, O., Baeza, I., Bombin, I., Bartrés-Faz, D., Arango, C., Gonzalez-Pinto, A., Parellada, M., Mayoral, M., Graell, M., Otero, S., Guardia, J., Castro- 
Fornieles, J., 2013. Cognitive reserve as a predictor of two year neuropsychological performance in early onset first-episode schizophrenia. Schizophr. Res. 143, 125-131.

de Oliveira, M.O., Nitrini, R., Yassuda, M.S., Brucki, S.M., 2014. Vocabulary is an appropriate measure of premorbid intelligence in a sample with heterogeneous educational level in Brazil. Behav. Neurol., 8759602014.

Esterberg, M., Compton, M., 2012. Family history of psychosis negatively impacts age at onset, negative symptoms, and duration of untreated illness and psychosis in first-episode psychosis patients. Psychiatry Res. 197 (1-2), 23-28.

First, M., Gibbon, M., Spitzer, R., Williams, J., Benjamin, L., 1997a. Structured Clinical Interview for DSM-IV Axis II Personality Disorders (SCID-II). American Psychiatric Publishing, Washington DC.

First, M., Spitzer, R., Gibbon, M., Williams, J., 1997b. Structured Clinical Interview for DSM-IV Axis I disorders-Clinician (SCID-I). American Psychiatric Press, Washington DC.

Fraley, C., Raftery, A.E., 1998. How many clusters? Which clustering method? Answers via model-based cluster analysis. Comput. J. 41 (8), 578-588.

Fusar-Poli, P., Nelson, B., Valmaggia, L., Yung, A.R., McGuire, P.K., 2014. Comorbid depressive and anxiety disorders in 509 individuals with an at-risk mental state: impact on psychopathology and transition to psychosis. Schizophr. Bull. 40 (1), 120-131.

Gardner, D., Murphy, A., O'Donnell, H., Centorrino, F., Baldessarini, R.J., 2010. International consensus study of antipsychotic dosing. Am. J. Psychiatry 167, 686-693.

Gardsjord, E.S., Romm, K.L., Friis, S., Barder, H.E., Evensen, J., Haahr, U., enVeldenHegelstad, W., Joa, I., Johannessen, O., Langeveld, J., Larse, T.K., Opjordsmoen, S., Rund, B.R., Simonsen, E., Vaglum, P., McGlashan, T., Melle, I., Røssberg, J.I., 2016. Subjective quality of life in first-episode psychosis. A ten year follow-up study. Schizophr. Res. 172 (1-3), 23-28.

González-Ortega, I., Rosa, A., Alberich, S., Barbeito, S., Vega, P., Echeburúa, E., Vieta, E., González-Pinto, A., 2010. Validation and use of the functioning assessment short test in first psychotic episodes. J. NervMent. Dis. 198 (11), 836-840.

Guy, W., 1976. ECDEU assessment manual for psychopharmacology, revised. In: US Department of Health, Education, and Welfare Publication (ADM). National Institute of Mental Health, Rockville, MD, pp. 76-338.

Hassani, M., Seidl, T., 2017. Using internal evaluation measures to validate the quality of diverse stream clustering algorithms. J. Comput. Sci. 4, 171-183.

Hinojosa-Marqués, L., Domínguez-Martínez, T., Kwapil, T.R., Barrantes-Vidal, N., 2019. Ecological validity of expressed emotion in early psychosis. Front. Psychiatry 10, 854.

Hollingshead, A., Redlich, F., 1958. Social class and mental illness: a community study. Am. J. Public Health 97, 1756-1757.

Jensen, J.H., Støttrup, M.M., Nayberg, E., Knorr, U., Ullum, H., Purdon, S.E., Kessing, L.V., Miskowiak, K.W., 2015. Optimising screening for cognitive dysfunction in bipolar disorder: validation and evaluation of objective and subjective tools. J. Affect. Disord. 187, 10-19.

Kay, S., Fiszbein, A., Opler, L., 1987. The positive and negative syndrome scale (PANSS) for schizophrenia. Schizophr. Bull. 13, 261-276.

Ketharanathan, T., Hanwella, R., Weerasundera, R., de Silva, V.A., 2015. Diagnostic validity and factor analysis of Montgomery-Asberg depression rating scale in Parkinson disease population. J. Geriatr. Psychiatry Neurol. 29 (3), 115-119.

Kirkpatrick, B., Strauss, G.P., Nguyen, L., Fischer, B.A., Daniel, D.G., Cienfuegos, A., Marder, S.R., 2011. The brief negative symptom scale: psychometric properties. Schizophr. Bull. 37, 300-305.

Kokkevi, A., Hartgers, C., 1995. European adaptation of a multidimensional assessment instrument for drug and alcohol dependence. Eur. Addict. Res. 1, 208-210.
Kring, A.M., Gur, R.E., Blanchard, J.J., Horan, W.P., Reise, S.P., 2013. The clinical assessment interview for negative symptoms (CAINS): final development and validation. Am. J. Psychiatry 170, 165-172.

Lee, G., Barrowclough, C., Lobban, F., 2013. Positive affect in the family environment protects against relapse in first-episode psychosis. Soc. Psychiatry Psychiatr. Epidemiol. 49, 367-376.

Lefort-Besnard, J., Varoquaux, G., Derntl, B., Gruber, O., Aleman, A., Jardri, R., Sommer, I., Thirion, B., Bzdok, D., 2018. Patterns of schizophrenia symptoms: hidden structure in the PANSS questionnaire. Transl Psychiatry 8 (1), 237.

Lobo, A., Chamorro, L., Luque, A., Dal-Ré, R., Badia, X., Baró, E., 2002. Validación de las versiones en español de la Montgomery-Asberg depression rating scale y la Hamilton anxiety rating scale para la evaluación de la depresión y de la ansiedad. Med. Clin. (Barc) 118 (13), 493-499.

Loo, R., 1979. The orthogonal rotation of factors in clinical research: a critical note. J. Clin. Psychol. 35, 762-765.

Malla, A., Payne, J., 2005. First-episode psychosis: psychopathology, quality of life, and functional outcome. Schizophr. Bull. 31 (3), 650-671.

Mezquida, G., Cabrera, B., Bioque, M., Amoretti, S., Lobo, A., González-Pinto, A., Espliego, A., Corripio, I., Vieta, E., CastroFornieles, J., Bergé, D., Escartí, M.J., Ibañez, Á., Penadés, R., Sánchez-Torres, A.M., Bernardo, M., 2017. The course of negative symptoms in first-episode schizophrenia and its predictors: a prospective two-year follow-up study. Schizophr. Res. 189, 84-90.

Montgomery, S., Asberg, M., 1979. A new depression scale designed to be sensitive to change. Br. J. Psychiatry 134, 382-389.

Moos, R.H., Moos, B.S., 1976. A typology of family social environments. Fam. Process. 15 (4), 357-371.

Norman, R., Malla, A., Manchanda, R., Harricharan, R., Takhar, J., Northcott, S., 2005. Social support and three-year symptom and admission outcomes for first episode psychosis. Schizophr. Res. 80, 227-234.

Peralta, V., Cuesta, M.J., 2001. How many and which are the psychopathological dimensions in schizophrenia? Issues influencing their ascertainment. Schizophr. Res. 49 (3), 269-285.

Perkins, D.O., Gu, H., Boteva, K., Lieberman, J.A., 2005. Relationship between duration of untreated psychosis and outcome in first-episode schizophrenia: a critical review and meta-analysis. Am. J. Psychiatry 162, 1785-1804.

Potuzak, M., Ravichandran, C., Lewandowski, K.E., Ongur, D., Cohen, B.M., 2012. Categorical vs dimensional classifcations of psychotic disorders. Compreh. Psychiatry 53 (8), 1118-1129.

Quilty, L.C., Robinson, J.J., Rolland, J.-.P., Fruyt, F.D., Rouillon, F., Bagby, R.M., 2013. The structure of the Montgomery-Åsberg depression rating scale over the course of treatment for depression. Int. J. Methods Psychiatr. Res. 22 (3), 175-184.

Rabinowitz, J., Harvey, P.D., Eerdekens, M., Davidson, M., 2006. Premorbid functioning and treatment response in recent-onset schizophrenia. Br. J. Psychiatry 189, 31-35.

Rocca, P., Fonzo, V., Ravizza, L., Rocca, G., Scotta, M., Zanalda, E., Bogetto, F., 2002. A comparison of paroxetine and amisulpride in the treatment of dysthymic disorder. J. Affect. Disord. 70, 313-317.

Rodriguez-Jimenez, R., Bagney, A., Mezquita, L., Martinez-Gras, I., Sanchez-Morla, E.M., Mesa, N., Ibañez, M., Diez-Martin, J., Jimenez-Arriero, M., Lobo, A., Santos, J., Palomo, T., 2013. Cognition and the five-factor model of the positive and negative syndrome scale in schizophrenia. Schizophr. Res. 143 (1), 77-83.

Rodríguez-Sánchez, J.M., Ayesa-Arriola, R., Pérez-Iglesias, R., Periañez, J.A., Martinez-Garcia, O., Gomez-Ruiz, E., Tabares-Seisdedos, R., Crespo-Facorro, B., 2013. Course of cognitive deficits in first episode of non-affective psychosis: a 3-year follow-up study. Schizophr. Res. 150, 121-128. 
Rosa, A.R., Sánchez-Moreno, J., Martínez-Aran, A., Salamero, M., Torrent, C., Reinares, M., Comes, M., Colom, F., Van Riel, W., Ayuso-Mateos, J.L., Kapczinski, F., Vieta, E., 2007. Validity and reliability of the functioning assessment short test (FAST) in bipolar disorder. Clin. Pract. Epidemiol. Ment. Health 7, 5.

Salagre, E., Arango, C., Artigas, F., Ayuso-Mateos, J.L., Bernardo, M., Castro-Fornieles, J., Bobes, J., Desco, M., Fañanás, L., González-Pinto, A., Haro, J.M., Leza, J.C., Mckenna, P.J., Meana, J.J., Menchón, J.J., Micó, J.A., Palomo, T., Pazos, Á., Pérez, V., Saiz-Ruiz, J., Sanjuán, J., Tabarés-Seisdedos, R., Crespo-Facorro, B., Casas, M., Vilella, E., Palao, D., Olivares, J.M., Rodriguez-Jimenez, R., Vieta, E., 2019. CIBERSAM: ten years of collaborative translational research in mental disorders. Rev. Psiquiatr. Salud. Ment. 12 (1), 1-8.

Salagre, E., Dodd, S., Aedo, A., Rosa, A., Amoretti, S., Pinzon, J., Reinares, M., Berk, M., Kapczinski, F.P., Vieta, E., Grande, I., 2018. Toward precision psychiatry in bipolar disorder: staging 2.0. Front. Psychiatry $9,641$.

Sim, K., Mahendran, R., Siris, S.G., Heckers, S., Chong, S.A., 2004. Subjective quality of life in first episode schizophrenia spectrum disorders with comorbid depression. Psychiatry Res. 129, 141-147.

Stein, D.J., Szatmari, P., Gaebel, W., Berk, M., Vieta, E., Maj, M., Anna de Vries, Y., Roest, A.M., de Jonge, P., Maercker, A., Brewin, C.R., Pike, K.M., Grilo, C.M., Fineberg, N.A., Briken, P., Cohen-Kettenis, P.T., Reed, G.M., 2020. Mental, behavioral and neurodevelopmental disorders in the ICD-11: an international perspective on key changes and controversies. BMC Med. 18 (1), 21.

Suganya, R., Shanthi, R., 2012. Fuzzy c-means algorithm-a review. IJSRP 2 (11), 1.

Suzuki, A., Aoshima, T., Fukasawa, T., Yoshida, K., Higuchi, H., Shimizu, T., Otani, K., 2005. A three-factor model of the MADRS in major depressive disorder. Depress. Anxiety 21, 95-97.

Tomasini, C., Borges, E.N., Machado, K., Emmendorfer, L., 2017. A study on the relationship between internal and external validity indices applied to partitioning and density-based clustering algorithms. In: Proceedings of the 19th International Conference on Enterprise Information Systems, 1, pp. 89-98 ICEIS 2017.
Tonna, M., Ossola, P., Marchesi, C., Bettini, E., Lasalvia, A., Bonetto, C., Lenzi, J., Rucci, P., Iozzino, L., Cellini, M., Comacchio, C., Cristofalo, D., D’Agostino, A., de Girolamo, G., De Santi, K., Ghigi, D., Leuci, E., Miceli, M., Meneghelli, A., Pileggi, F., Scarone, S., Santonastaso, P., Torresani, S., Tosato, S., Veronese, A., Fioritti, A., Ruggeri, M., Group, GET UP, 2019. Dimensional structure of first episode psychosis. Early Interv. Psychiatry 13 (6), 1431-1438.

Uher, R., Farmer, A., Maier, W., Rietschel, M., 2008. Measuring depression: comparison and integration of three scales in the GENDEP study. Psychol. Med. 38, 289-300.

Van Os, J., Kapur, S., 2009. Schizophrenia. Lancet. 374, 635-645.

Vares, F., Smeets, F., Drukker, M., Lieverse, R., Lataster, T., Viechtbauer, W., Read, J., van Os, J., Bentall, R.P., 2012. Childhood adversities increase the risk of psychosis: a meta-analysis of patient-control, prospective- and cross-sectional cohort studies. Schizophr. Bull. 38 (4), 661-671.

Verma, S., Poon, L.Y., Subramaniam, M., Abdin, E., Chong, S.A., 2012. The Singapore early psychosis intervention programme (EPIP): a programme evaluation. Asian J. Psychiatr. 5 (1), 63-67.

Vieta, E., 2016. DSM-5.1. Acta Psychiatr. Scand. 134 (3), 187-188.

White, C., Stirling, J., Hopkins, R., Morris, J., Montague, L., Tantam, D., Lewis, S., 2009. Predictors of 10-year outcome of first episode psychosis. Psychol. Med. 39, 1447-1456.

Williamson, D., Brown, E., Perlis, R.H., Ahl, J., Baker, R.W., Tohen, M., 2006. Clinical relevance of depressive symptom improvement in bipolar I depressed patients. J. Affect. Disord. 92, 261-266.

Organization, World Health, 2004. ICD-10: International Statistical Classification of Diseases and Related Health problems: Tenth Revision, 2nd ed. World Health Organization.

Wu, C., Luedtke, A.R., Sadikova, E., Tsai, H., Liao, S., Liu, C., Gau, S.S., VanderWeele, T.J., Kessler, R.C., 2020. Development and validation of a machine learning individualized treatment rule in first-episode schizophrenia. JAMA Netw. Open 3 (2), e1921660.

Wunderink, L., van Bebber, J., Sytema, S., Boonstra, N., Meijer, R.R., Wigman, J.T.W., 2020. Negative symptoms predict high relapse rates and both predict less favorable functional outcome in first episode psychosis, independent of treatment strategy. Schizophr. Res. 216, 192-199. 